\title{
Ataxin-3 Represses Transcription via Chromatin Binding, Interaction with Histone Deacetylase 3, and Histone Deacetylation
}

\author{
Bernd O. Evert, ${ }^{1}$ Julieta Araujo, ${ }^{1}$ Ana M. Vieira-Saecker, ${ }^{1}$ Rob A. I. de Vos, ${ }^{2}$ Sigrid Harendza, ${ }^{3}$ Thomas Klockgether, ${ }^{1}$ \\ and Ullrich Wüllner ${ }^{1}$ \\ ${ }^{1}$ Department of Neurology, University of Bonn, 53105 Bonn, Germany, ${ }^{2}$ Laboratorium Pathologie Oost Nederland, 7512 AD Enschede, The Netherlands, \\ and ${ }^{3}$ Department of Nephrology, University of Hamburg, 20246 Hamburg, Germany
}

\begin{abstract}
Ataxin-3 (AT3), the disease protein in spinocerebellar ataxia type 3 (SCA3), has been associated with the ubiquitin-proteasome system and transcriptional regulation. Here we report that normal AT3 binds to target DNA sequences in specific chromatin regions of the matrix metalloproteinase-2 (MMP-2) gene promoter and represses transcription by recruitment of the histone deacetylase 3 (HDAC3), the nuclear receptor corepressor ( $\mathrm{NCoR}$ ), and deacetylation of histones bound to the promoter. Both normal and expanded AT3 physiologically interacted with HDAC3 and NCoR in a SCA3 cell model and human pons tissue; however, normal AT3-containing protein complexes showed increased histone deacetylase activity, whereas expanded AT3-containing complexes had reduced deacetylase activity. Consistently, histone analyses revealed an increased acetylation of total histone H3 in expanded AT3-expressing cells and human SCA3 pons. Expanded AT3 lost the repressor function and displayed altered DNA/chromatin binding that was not associated with recruitment of HDAC3, NCoR, and deacetylation of the promoter, allowing aberrant MMP-2 transcription via the transcription factor GATA-2. For transcriptional repression normal AT3 cooperates with HDAC3 and requires its intact ubiquitin-interacting motifs (UIMs), whereas aberrant transcriptional activation by expanded AT3 is independent of the UIMs but requires the catalytic cysteine of the ubiquitin protease domain. These findings demonstrate that normal AT3 binds target promoter regions and represses transcription of a GATA-2dependent target gene via formation of histone-deacetylating repressor complexes requiring its UIM-associated function. Expanded AT3 aberrantly activates transcription via its catalytic site and loses the ability to form deacetylating repressor complexes on target chromatin regions.
\end{abstract}

Key words: ataxin-3; polyglutamine; histone acetylation; HDAC3; NCoR; MMP-2

\section{Introduction}

Spinocerebellar ataxia type 3 (SCA3) is caused by an unstable CAG repeat expansion in the SCA3 gene leading to an expansion of polyglutamines in the corresponding protein, ataxin-3 (AT3) (Kawaguchi et al., 1994), and belongs to the group of polyglutamine (polyQ) diseases (Zoghbi and Orr, 2000). An important aspect of polyQ diseases is that the non-expanded protein localizes to nuclear inclusions (NIs) formed by the expanded protein (Uchihara et al., 2001; Haacke et al., 2006). Therefore, both specific features of the mutant and the loss of function of the normal protein may contribute to pathology.

Several lines of evidence demonstrate that AT3 is involved in the ubiquitin-proteasome (UPS) system. AT3 binds polyubiq-

Received May 13, 2006; revised Aug. 27, 2006; accepted Sept. 21, 2006.

This work was supported by the Deutsche Forschungsgemeinschaft, a University of Bonn Center Grant, and FP6 Contract LSHM-CT-2004-503304 from European Union. We thank Dr. Yvon Trottier and Dr. Erich Wanker for kindly providing the anti-ataxin-3 antibodies. We are thankful to Dr. Lucia Ozimek for performing the coimmunofluorescence stainings.

Correspondence should be addressed to Dr. Bernd Evert, Department of Neurology, University of Bonn, SigmundFreud-Strasse 25, 53105 Bonn, Germany. E-mail: b.evert@uni-bonn.de.

DOI:10.1523/JNEUROSCI.2053-06.2006

Copyright $\odot 2006$ Society for Neuroscience $\quad$ 0270-6474/06/2611474-13\$15.00/0 uitin via its ubiquitin-interacting motifs (UIMs) (Burnett et al., 2003; Donaldson et al., 2003; Chai et al., 2004). AT3 is a ubiquitin-specific protease (Amerik and Hochstrasser, 2004; Mao et al., 2005; Nicastro et al., 2005) and is involved in the recognition of proteolytic substrates by the proteasome (DossPepe et al., 2003; Berke et al., 2005). Moreover, AT3 regulates aggresome formation (Burnett and Pittman, 2005) and suppresses polyQ-induced toxicity via its ubiquitin-dependent activities (Warrick et al., 2005).

Another line of evidence suggests that AT3 acts as a transcriptional repressor via inhibition of histone acetyltransferase (HAT) activity of the major transcriptional coactivators, cAMP response element-binding protein (CREB) binding protein (CBP), p300, and p300/CBP-associated factor (PCAF) (Li et al., 2002). Transcriptional coactivators with HAT activity promote histone acetylation to achieve transcriptional activation (Torchia et al., 1997). In contrast, deacetylation of histones via histone deacetylases (HDACs) renders gene promoters inaccessible by regulatory factors (Nagy et al., 1997). The altered balance between histone acetylation and deacetylation may be a key process contributing to polyQ-induced pathogenesis. For instance, huntingtin interacts with the nuclear receptor corepressor $(\mathrm{NCoR})$ and $\operatorname{Sin} 3 \mathrm{~A}$ 
(Boutell et al., 1999; Steffan et al., 2000; Kegel et al., 2002), ataxin-7 inhibits the acetylation activity of TATA-binding protein (TBP)-free TBP-associated factor (TAF)-containing complex and SPT3/TAF9/GCN5 acetyltransferase complex (Strom et al., 2005), and ataxin-1 inhibits transcription via (1) interaction with the corepressor silencing mediator of retinoic acid and thyroid hormone receptor (SMRT) and HDAC3 (Tsai et al., 2004) and (2) downregulation of transcriptional coregulators interacting with the AXH domain of ataxin-1 (de Chiara et al., 2005; Tsuda et al., 2005).

We previously identified differentially expressed genes in a SCA3 cell model and human SCA3 brain (Evert et al., 2001, 2003). Several genes, among them the matrix metalloproteinase-2 (MMP-2) gene, were downregulated by normal AT3 but upregulated by expanded AT3, suggesting a role for AT3 in transcriptional repression that is no longer maintained by the expanded protein. The loss of the normal protein function, i.e., repression of gene expression, therefore, simultaneously results in a gain of function via aberrantly activated gene expression. Here we elucidate the molecular basis of this dual effect and show that normal AT3 binds specific promoter regions of the MMP-2 gene and mediates transcriptional repression by interaction with HDAC3 and NCoR and deacetylation of histones bound to the MMP-2 promoter. Expanded AT3 failed to recruit HDAC3 and NCoR to specific chromatin regions, allowing increased binding of the transcription factor GATA-2 and increased transcription of MMP-2.

\section{Materials and Methods}

SCA3 cell model and human brain tissue. The SCA3 cell model comprises several stably transfected rat mesencephalic CSM14.1 clonal cell lines (SCA3 cell lines) expressing no transgene (Ctrl), normal (Q23), or expanded (Q70) human full-length AT3 (Evert et al., 1999). The cell lines, originally generated as tetracycline-responsive cell lines, provide a high level of expression of the recombinant AT3 isoforms (except the Ctrl cell lines) without tetracycline; the addition of tetracycline does not inhibit expression of the recombinant AT3 isoforms. All experiments with the SCA3 cell lines were performed without tetracycline at the permissive temperature $\left(33^{\circ} \mathrm{C}\right)$, as previously described (Evert et al., 1999). The SCA3 brain tissues were derived from two patients with genetically confirmed diagnosis of SCA3 (one female of 59 years and one male of 62 years); two unaffected individuals (two males of 51 and 64 years) without a history of neurological illness served as the control.

Reporter and expression constructs. The MMP-2 gene promoter reporter constructs were generated in the promoter-less luciferase reporter vector pGL2-Basic (Promega, Madison, WI). Reporter constructs T6, T7, T8, T9, T10, and T11 were generated by PCR amplification, using KpnI and BglII flanked primers and subcloning into the KpnI and BglII sites of pGL2 vector. Reporter constructs T1, T2, T3, T4, and T5 were generated by PCR amplification, using KpnI flanked primers and subcloning into the KpnI site of reporter construct T6. PCR amplifications were performed by using genomic DNA of native CSM14.1 cells and the primers listed in supplemental Table 1 (available at www.jneurosci.org as supplemental material). Mutagenesis of the consensus binding sites for GATA-2 in T6 was performed by site-directed mutagenesis (QuikChange, Stratagene, La Jolla, CA) and primers replacing the conserved nucleotides of the binding sites (supplemental Table 2, available at www.jneurosci.org as supplemental material). MMP-2 primers correspond to the rat MMP-2 gene with the GenBank accession number DQ915967.

Expression constructs encoding full-length human normal or expanded AT3 were generated by subcloning the SCA3 cDNA BamHI inserts of the previously described response plasmids pUHD-SCA3-Q23 and pUHD-SCA3-Q70 (Evert et al., 1999) into pcDNA3 (Invitrogen, Carlsbad, CA), resulting in expression constructs pcDNA-AT3Q23 and pcDNA-AT3Q70. These constructs were used additionally to generate the C14A, L229/249A, and C14A-L229/249A mutants of AT3Q23 and
AT3Q70 by site-directed mutagenesis, with primers replacing the respective amino acids at the indicated amino acid positions (supplemental Table 2, available at www.jneurosci.org as supplemental material). The eukaryotic HDAC3 expression vector was obtained from InvivoGen (San Diego, CA). The N-terminal His-tagged AT3Q23, AT3Q70, and HDAC3 constructs for bacterial expression were generated by subcloning the $\mathrm{BamHI}$ inserts from the respective eukaryotic expression vectors into pQE30 (Qiagen, Hilden, Germany). Glutathione S-transferase-AT3 (GST-AT3) fusion constructs for bacterial expression were generated by subcloning the above SCA3 cDNA BamHI inserts into pGEX-5X-1 (Amersham Biosciences, Braunschweig, Germany). All constructs were verified by sequencing and expression analysis.

Reporter assays. For reporter assays, $1.5 \times 10^{5}$ cells of the stable SCA3 cell lines (Ctrl, Q23, and Q70) or native CSM14.1 cells were seeded on 24 -well plates. Cells were transfected the next day with $200 \mathrm{ng}$ of the indicated reporter constructs by FuGENE 6 (Roche, Palo Alto, CA) according to the instructions of the manufacturer. The reporter constructs always were cotransfected with $0.4 \mathrm{ng}$ of the coreporter Renilla luciferase (pRL-CMV, Promega). After $72 \mathrm{~h}$ luciferase activities were measured by the dual-luciferase reporter assay system (Promega) according to the instructions of the manufacturer and by a microplate luminometer (Berthold, Bad Wildbad, Germany). Data were normalized for activity of Renilla luciferase to account for transfection efficiency. Results from four independent experiments, each with $n=4$, were averaged and presented as the mean $\pm \mathrm{SD}$.

Chromatin immunoprecipitation assays. Chromatin immunoprecipitation (ChIP) assays were performed by using the ChIP-IT kit (Active Motif, Rixensart, Belgium), following the instructions of the manufacturer. In brief, $5 \times 10^{6}$ cells of each SCA3 cell line (Ctrl, Q23, and Q70) were grown on $10 \mathrm{~cm}$ plates to $80 \%$ confluency. Cells were fixed by adding formaldehyde ( $1 \%$ final concentration), and cross-linked adducts were resuspended and sonicated, resulting in an average chromatin fragment size of $400 \mathrm{bp}$. For ChIP assays rabbit polyclonal antibodies were used against AT3 [N-terminal region of the Machado-Joseph disease protein (NT-MJD), provided by Dr. Erich Wanker, Max-Delbrueck Center for Molecular Medicine, Berlin, Germany], GATA-2 and NCoR (Santa Cruz Biotechnology, Santa Cruz, CA), HDAC3 and control IgG (Active Motif), and acetyl-histone $\mathrm{H} 3$ and H4 (Upstate Biotechnology, Lake Placid, NY). Protein-bound immunoprecipitated DNA was reverse cross-linked and purified by using DNA purification mini-columns. The primers used for amplification of chromatin fragments of the MMP-2 gene (GenBank accession number DQ915967) are listed in supplemental Table 3 (available at www.jneurosci.org as supplemental material). The amount of immunoprecipitated chromatin DNA was normalized to the amount of input chromatin DNA determined by densitometric quantification of the generated PCR products. Input represents $10 \%$ of chromatin DNA used for immunoprecipitation. Values are expressed as fold enrichment over background immunoprecipitation signals obtained in corresponding control IgG antibody ChIP assays.

Transfection, RNA isolation, and PCR analysis. Total RNA was prepared as previously described (Evert et al., 2001) from the stable SCA3 cell lines (Ctrl, Q23, and Q70) after 7 d or from native CSM14.1 cells transiently transfected with the indicated constructs after $72 \mathrm{~h}$. For transient transfection of native CSM14.1 cells, $3.0 \times 10^{6}$ cells were seeded on $10 \mathrm{~cm}$ plates and transfected with FuGENE 6 (Roche) and $3 \mu \mathrm{g}$ of the indicated constructs. Synthesis of cDNA and PCR amplification were performed as previously described (Evert et al., 2003) with the primers listed in supplemental Table 4 (available at www.jneurosci.org as supplemental material).

ChIP-based cloning, DNA binding site search, and electrophoretic mobility shift assays. ChIP is a validated method to identify site-specific interactions of transcription factors and promoter DNA in the context of a living cell (Weinmann, 2004). To identify potential DNA binding sites of AT3 within the MMP-2 promoter and unknown AT3-bound target genes, we generated a chromatin library of Q23 cells by using a modified ChIP-based cloning approach (Huang et al., 2006). Briefly, chromatin from Q23 cells was precipitated with the AT3-antibody (NT-MJD) and digested with $\mathrm{MboI}$. The digested chromatin was reverse cross-linked, purified, subcloned into a BamHI-digested pUC18 vector and used to 
generate a library of 980 clones containing AT3-bound chromatin fragments. Forty-four clone inserts were sequenced and identified by BLAST (basic local alignment search tool) search at the National Center for Biotechnology Information (Bethesda, MD). Twenty inserts matched to promoters and coding regions of annotated rat genes (supplemental Table 5, available at www.jneurosci.org as supplemental material), whereas the remaining represented unknown regions of the rat genome or showed low similarity $(<10 \%)$ to annotated rat genes. Pairwise and multiple alignments with CLUSTAL W (European Molecular Biology Laboratory/European Bioinformatics Institute) and DiAlign (Genomatix Software, München, Germany) were used to search for recurring sequence elements on either DNA strand among the annotated gene fragments. Previously, transcriptional regulatory sequences of 6 bp length found in $>23 \%$ within a list of coordinately regulated genes have been used successfully to predict potential transcriptional regulatory sequences (Cho et al., 1998). Sixteen 6 bp sequences were identified and assigned to six categories of potential AT3 core motifs. The selected $6 \mathrm{bp}$ sequences of each category displayed exact matches on either DNA strand in at least $30 \%$ of the 20 searched and 44 sequenced AT3-bound genomic fragments (supplemental Table 5, available at www.jneurosci. org as supplemental material) and were found at least once in the MMP-2 promoter sequence. Complementary 17-20 bp oligonucleotide probes (supplemental Table 6, available at www.jneurosci.org as supplemental material) representative for each identified potential AT3 regulatory $6 \mathrm{bp}$ sequence were selected from the MMP-2 gene promoter (GenBank accession number DQ915967) and tested for binding to recombinant AT3Q23 and AT3Q70 in electrophoretic mobility shift assays (EMSAs). EMSAs were performed as previously described (Schmitt et al., 2003), using the indicated protein amounts of purified recombinant His-tagged AT3Q23, AT3Q70, and HDAC3. For supershift and competition assays 2 $\mu \mathrm{g}$ of the AT3 antibody (NT-MJD) or an excess of the respective unlabeled double-stranded oligonucleotides was preincubated with the indicated His-tagged proteins for $1 \mathrm{~h}$ at $4^{\circ} \mathrm{C}$. After addition of the respective labeled probe and incubation for $20 \mathrm{~min}$ on ice, DNA-protein complexes were separated on $8 \%$ nondenaturating polyacrylamide gels $(0.5 \times$ Trisborate EDTA) at $350 \mathrm{~V}$. Gels were vacuum-dried and exposed to x-ray films with intensifying screens.

Gelatin zymography. Zymography analysis of secreted proteolytically active MMP-2 protein was performed as described previously (Evert et al., 2001). Serum-containing medium of transiently transfected native CSM14.1 cells was aspirated $48 \mathrm{~h}$ after transfection and replaced with serum-free medium and additionally incubated for $24 \mathrm{~h}$. After collecting and concentrating the medium, we separated $50 \mu \mathrm{g}$ of protein of the concentrated medium in $10 \%$ polyacrylamide gels with gelatin. Gels then were developed and stained as described previously (Evert et al., 2001).

Nuclear cell and brain extracts. Nuclear extracts of SCA3 cell lines were prepared as previously described (Murphy et al., 2001). Briefly, $3 \times 10^{6}$ cells of each SCA3 cell line (Ctrl, Q23, and Q70) were grown on $10 \mathrm{~cm}$ plates. After $7 \mathrm{~d}$ the cells were collected and resuspended in buffer A [containing the following (in mM): 10 HEPES, pH 7.9, $1.5 \mathrm{MgCl}_{2}, 10$ $\mathrm{NaCl}, 0.5 \mathrm{DTT}$, and $0.1 \% \mathrm{NP}-40$ ] containing $0.1 \%$ Triton X-100. After centrifugation at $2000 \times g$ the nuclear pellet was resuspended in buffer $\mathrm{B}$ [containing the following (in mM): 20 HEPES, pH 7.9, $1.5 \mathrm{MgCl}_{2}, 0.2$ EDTA, plus $0.42 \mathrm{M} \mathrm{NaCl}$ and $25 \%$ glycerol]. After centrifugation at $12,000 \times g$ the resulting supernatant represented the nuclear extract. Both buffers contained complete protease inhibitor mixture (Roche). Total human brain extracts were prepared from fresh-frozen postmortem pons tissue of controls and SCA3 patients. Tissue $(100 \mathrm{mg})$ was homogenized in radioimmunoprecipitation assay (RIPA) buffer [containing the following (in mM): $150 \mathrm{NaCl}, 50$ Tris- $\mathrm{HCl}, \mathrm{pH} 8.0$, plus $1 \%$ NP-40, $0.5 \%$ DOC (deoxycholic acid), $0.1 \%$ SDS] containing complete protease inhibitor mixture (Roche) by using an Ultra-Turrax Disperser (IKA, Staufen, Germany). Extracts were clarified by centrifugation at $12,000 \times g$.

Histone extraction. For histone extraction $3.0 \times 10^{6}$ cells were seeded on $10 \mathrm{~cm}$ plates and incubated for $7 \mathrm{~d}$. Harvested cells were resuspended in Triton extraction buffer [PBS containing 0.5\% Triton X-100, $2 \mathrm{~mm}$ phenylmethylsulfonyl fluoride (PMSF), $0.02 \% \mathrm{NaN}_{3}$, and $5 \mathrm{~mm}$ sodium butyrate]. After centrifugation at $400 \times g$, the pellet was resuspended in
Triton extraction buffer and centrifuged again. The pellet was resuspended in $0.2 \mathrm{~N} \mathrm{HCl}$, and histones were acid-extracted overnight at $4^{\circ} \mathrm{C}$. After centrifugation at $400 \times g, 50 \mu \mathrm{g}$ of protein of the supernatant was separated in $15 \%$ polyacrylamide gels and analyzed by Western blot. From fresh-frozen postmortem pons tissue of controls and SCA3 patients $50 \mathrm{mg}$ was homogenized in Triton extraction buffer with an UltraTurrax Disperser (IKA), and histones were extracted as described above.

Immunoprecipitations and Western blot analysis. For immunoprecipitation $400 \mu \mathrm{g}$ of protein of SCA3 cell nuclear extracts or human brain extracts was incubated with the indicated antibody $(2 \mu \mathrm{g})$, followed by incubation with protein G-Sepharose beads (Amersham Biosciences). Beads were washed with RIPA buffer, boiled in $2 \times$ Laemmli loading buffer, and separated by SDS-gel electrophoresis. The antibodies used for immunoprecipitation were anti-AT3 (NT-MJD), anti-HDAC3 (Active Motif) and anti-NCoR and rabbit control IgG (Santa Cruz Biotechnology). Western blot analysis was performed as previously described (Evert et al., 1999), using in addition the antibodies mouse monoclonal antiAT3 (1H9, provided by Dr. Yvon Trottier, Institut de Genetique et de Biologie Moleculaire et Cellulaire, Illkirch, France), anti-ubiquitin (Biotrend, Köln, Germany), anti-actin (against all isoforms) (Sigma, München, Germany), rabbit polyclonal anti-histone H3 (Upstate Biotechnology), anti-ubiquitin (BostonBiochem, Cambridge, MA), and goat polyclonal anti-GST (Amersham Biosciences).

Protein expression and in vitro binding experiments. His-tagged HDAC3 was expressed in Escherichia coli M15 and purified on Ni-NTA (nickel-nitrilotriacetic) affinity columns according to the instructions of the manufacturer (Qiagen). GST-AT3 fusion proteins were expressed in E. coli BL21 and purified with glutathione-Sepharose 4B beads according to the instructions of the manufacturer (Amersham Biosciences). The eluted GST-AT3 fusion proteins were incubated with purified HisHDAC 3 or $400 \mu \mathrm{g}$ of protein of Ctrl cell nuclear extracts in RIPA buffer at room temperature for $1 \mathrm{~h}$. Then glutathione-Sepharose beads were added, and incubation was continued at $4^{\circ} \mathrm{C}$ overnight with rotation. Beads were washed with RIPA buffer, boiled in $2 \times$ Laemmli loading buffer, and analyzed by Western blot.

Immunofluorescence. Coimmunofluorescence staining was performed as described previously (Evert et al., 2001, 2003) with formalin-fixed pons sections from controls and SCA3 patients by using the anti-AT3 (1H9) antibody (1:1500) together with anti-HDAC3 (1:400) and antiNCoR (1:400) antibody, followed by Texas Red goat anti-mouse and fluorescein goat anti-rabbit antibodies (Jackson ImmunoResearch, West Grove, PA). Samples were viewed with a Nikon Eclipse E800 fluorescence microscope (Nikon, Düsseldorf, Germany). Digitized images were collected on separate fluorescence channels with a Sony 3CCD digital camera.

HDAC activity assays. HDAC activity was measured with immunoprecipitated protein complexes from SCA3 cell nuclear extracts by a fluorescent assay kit (FluordeLys, Biomol, Plymouth Meeting, PA). In brief, the immunoprecipitated beads were prepared and washed as described above and resuspended in $60 \mu \mathrm{l}$ of HDAC buffer [containing the following (in mM): 50 Tris- $\mathrm{HCl}, \mathrm{pH} 8.0,137 \mathrm{NaCl}, 2.7 \mathrm{KCl}$, and $1 \mathrm{MgCl}_{2}$ ]. The assay was conducted at room temperature according to the manufacturer's protocol, using one-third of the bead suspension. The samples were prepared in triplicate, and the fluorescence was measured on a microplate fluorescence reader (Molecular Devices, Palo Alto, CA).

Statistics. Statistical analysis was performed via one-way ANOVA, followed by Tukey-Kramer multiple comparisons test. Data are expressed as the mean $\pm \mathrm{SD}$.

\section{Results \\ RE2 mediates transcriptional activation and repression of MMP-2 in SCA3 cell lines}

Compared with stably mock-transfected rat mesencephalic CSM14.1 cells (Ctrl), MMP-2 gene transcription is repressed in CSM14.1 cell lines stably expressing normal human full-length AT3 with 23 glutamines (Q23) but is increased in cell lines expressing expanded AT3 with 70 glutamines (Q70) (Fig. 1, inset) (Evert et al., 2001, 2003). To identify promoter regions of the 
A
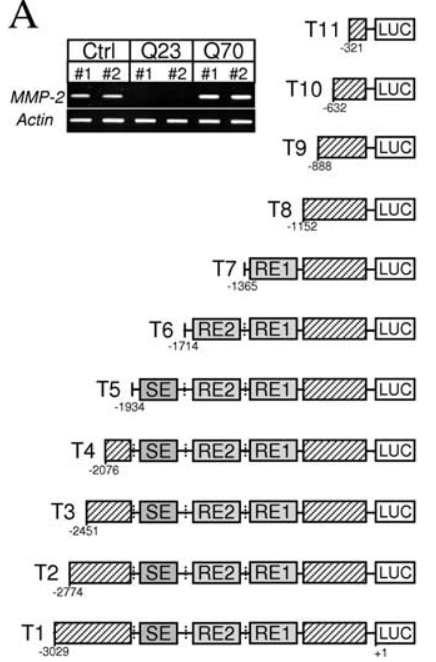

B

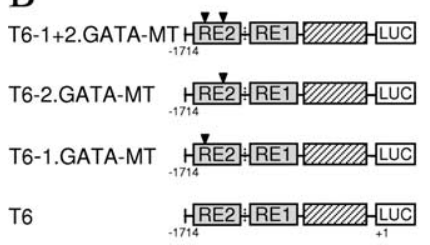

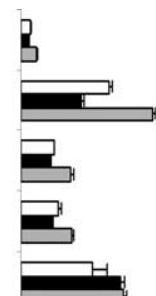
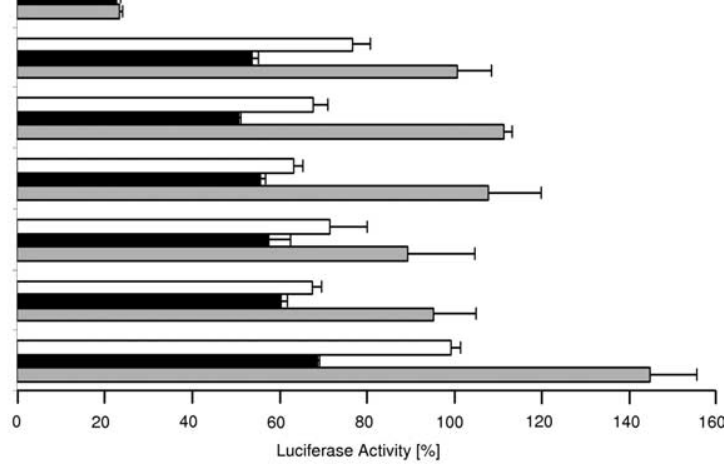

Luciferase Activity [\%]

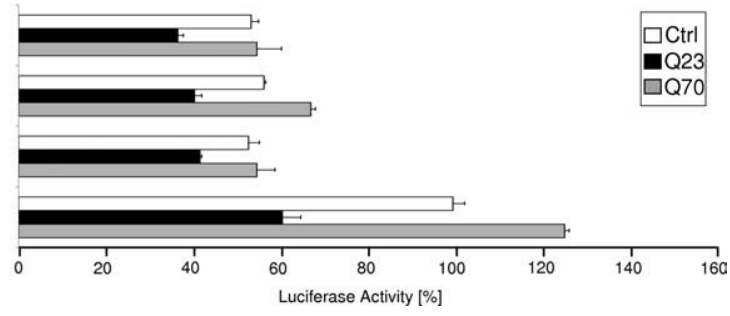

Figure 1. MMP-2 gene transcription in SCA3 cell lines is activated via GATA-2 consensus sites within the enhancer region RE2 of the MMP-2 promoter. Inset, Semiquantitative PCR analysis for endogenous MMP-2 and actin expression, using CDNA from individual clonal (\#1 and \#2) SCA3 cell lines (Ctrl, Q23, and Q70). A, Reporter assays of SCA3 cell lines transfected with constructs containing the entire known proximal MMP-2 gene promoter (T1) and serial truncations thereof down to -321 (T2 to T11). Luciferase (LUC) reporter gene constructs are shown at left schematically, together with their respective truncation site in relation to the translational start site $(+1)$ of the MMP-2 gene. Known enhancer (RE1 and RE2) and silencer (SE) regions are shown in gray and truncated regions in hatched boxes. Three promoter regions within reporter constructs T10 (region - 632 to -321), T6 (region -1714 to -1365 ), and T1 (region -3029 to -2774) mediated normal or strong transcriptional activation in Ctrl and Q70 cells, respectively, whereas these regions concurrently were repressed in Q23 cells. The luciferase activities are presented as a percentage of activity measured in Ctrl cells transfected with construct $\mathrm{T1}$. The results were averaged from four independent experiments $(n=4)$ and are presented as the mean \pm SD. $\boldsymbol{B}$, Reporter assays of SCA3 cell lines with the wild-type promoter construct T6 and analogous T6 promoter constructs containing each (T6-1.GATA-MT, T6-2.GATA-MT) or both GATA-2 sites mutated (T61+2.GATA-MT). Inverted triangles indicate the mutated binding sites. Mutagenesis of each or both GATA-2 binding sites within construct T6 strongly reduced the activation of MMP- 2 in Ctrl and Q70 cells and additionally repressed activity in Q23 cells. The luciferase activities are presented as a percentage of activity measured in Ctrl cells transfected with wild-type construct T6. The results were averaged from four independent experiments $(n=4)$ and are presented as the mean \pm SD.

MMP-2 gene mediating transcriptional repression or activation in SCA3 cell lines, we generated luciferase reporter gene constructs containing the entire known proximal region and serial truncations of the rat MMP-2 gene promoter (T1-T11) and transiently transfected them into the SCA3 cell lines (Fig. 1A). The strongest transcriptional activation in SCA3 cell lines was obtained with construct $\mathrm{T} 6$ containing the previously identified enhancer region 2 (RE2) (Han et al., 2003). Ctrl and Q70 cells showed a fivefold increase, whereas Q23 cells showed only a twofold increase of luciferase activities when compared with T7 (Fig. $1 A$ ). Two additional promoter regions within construct $\mathrm{T} 10$ and the full-length construct $\mathrm{T} 1$ showed increased transcriptional activation in Ctrl and Q70 cells as compared with moderate increases in Q23 cells (Fig. 1A). Thus three promoter regions within reporter constructs T10 (region -632 to -321), T6 (region -1714 to -1365 ) and T1 (region -3029 to -2774 ) were activated transcriptionally in SCA3 cell lines. The same regions of the MMP-2 promoter were targeted for repression in Q23 cells, be- cause the extent of activation is always lower in Q23 as compared with Ctrl and Q70 cells.

RE2 contains two predicted consensus sites for the transcription factor GATA-2 (Han et al., 2003). Mutagenesis of each GATA-2 binding site within construct T6 strongly reduced transcriptional activation of MMP-2 in Ctrl and Q70 cells and additionally repressed activity in Q23 cells (T6-1.GATA-MT and T6-2.GATA-MT) (Fig. 1B). Combined mutagenesis of both sites did not reduce activity additionally $($ T6-1+2.GATA-MT) (Fig. 1B). These findings show that both GATA-2 consensus sites in RE2 are required for the upregulation of MMP-2 in SCA3 cell lines and indicated that the repressing mechanism observed in Q23 cells could affect the GATA-2-dependent transcriptional activation.

\section{HDAC 3 and AT3 are recruited to transcriptionally repressed regions in Q23 cells}

GATA-2 transcriptional activity is repressed by HDAC3 via direct interaction (Ozawa et al., 2001). This interaction is proposed to result in recruitment of HDAC3 to GATA-2 target promoters, deacetylation of target chromatin, and repression of GATA-2-dependent target genes. We thus asked whether the transcriptional repression of MMP-2 in Q23 cells correlates with a reduced binding of GATA-2, enhanced recruitment of HDAC3, and histone deacetylation of the MMP-2 promoter. ChIP assays were performed by using cross-linked chromatin from the SCA3 cell lines and specific antibodies against HDAC3, GATA-2, and acetylated histone $\mathrm{H} 3$ and $\mathrm{H} 4$ to analyze the complete known region of the MMP-2 promoter divided into 11 adjacent amplicons (A1-A11) of $\sim 300 \mathrm{bp}$ in length

(Fig. 2A).

Q23 cells showed strongly increased HDAC3 recruitment throughout the distal promoter region, with the highest enrichment (14- to 16-fold) in regions corresponding to amplicons A1, A4, A6, and A10 (Fig. 2 B). In Ctrl and Q70 cells these promoter regions showed no increased HDAC3 recruitment but instead revealed an enrichment of GATA-2-bound fragments in A1, A6, and A10 (Fig. 2C). In Q70 the highest enrichment of GATA-2bound chromatin was found in A6 (15-fold), in agreement with the GATA-2-dependent transcriptional activation of RE2 in the reporter assays (Fig. 1). Consistent with HDAC3-mediated repression of GATA-2, Q23 cells showed reduced GATA-2 binding in these regions (Fig. 2C). In agreement with a HDAC3-mediated histone deacetylation and transcriptional repression of the MMP-2 promoter, Q23 cells revealed reduced levels of acetylated histone $\mathrm{H} 3$ in HDAC3-bound and all other promoter regions (Fig. 2E). Ctrl and Q70 cells, instead, showed $>10$ - and 15-fold increased levels of acetylated histone $\mathrm{H} 3$ in promoter regions $\mathrm{A} 1$, 
A5, A6, and A10 (Fig. 2 E). An increase of acetylated $\mathrm{H} 4$ ( $>10$-fold) was found only in Q70 cells in A1 and A6 (Fig. 2G). The increased histone acetylation in Q70 cells reflects the transcriptionally active MMP-2 promoter and the reduced acetylation of histones in Q23cells the transcriptionally repressed MMP-2 promoter found in the reporter assays.

We additionally investigated whether AT3 itself binds to the MMP-2 promoter, because AT3 has been shown to bind histones in vitro (Li et al., 2002). ChIP assays showed an enrichment (8- to 12-fold) of AT3-bound chromatin fragments in A1, A4, A6, and A10 (Fig. 2D) that closely corresponded to the HDAC-3-bound regions in Q23 cells, revealing that AT3 binds chromatin and could be involved in transcriptional repression of MMP-2. In contrast, Q70 cells showed an increased AT3 binding (7- to 10-fold) to promoter regions in $\mathrm{A} 2$ and $\mathrm{A} 5$ (Fig. 2D), indicating an altered binding of AT3 that is not associated with the recruitment of HDAC3. HDAC3 requires the transcriptional corepressor NCoR to exhibit HDAC activity (Li et al., 2000; Guenther et al., 2001). Therefore, we additionally analyzed whether NCoR is recruited to HDAC3and AT3-bound chromatin regions. ChIP assays of the MMP-2 promoter in Q23 cells confirmed a 10- to 12 -fold enrichment of NCoR in the same AT3- and HDAC3-bound regions (A1, A4, A6, and A10), whereas Ctrl and Q70 cells showed weak (A1, A6, and A10) or no enrichment of NCoR, respectively, in these regions (Fig. 2 F). Collectively, these findings demonstrate that the transcriptional repression of MMP-2 in Q23 cells is associated with recruitment of AT3, HDAC3, and NCoR, deacetylation of core histones, and reduced binding of GATA-2 to target regions of the MMP-2 promoter.

\section{AT3 binds to specific DNA sequences of the MMP-2 promoter}

To reveal whether chromatin targeting by AT3 involves direct binding to DNA and to search for potential binding sequences of AT3, we generated a library by subcloning AT3-bound chromatin fragments from Q23 cells and aligned the retrieved annotated genomic sequences to search for recurring motifs (see Materials and Methods). Alignments revealed six candidate categories of core motifs with $6 \mathrm{bp}$ sequences occurring in at least $30 \%$ of the analyzed clones (supplemental Table 5, available at www.jneurosci.org as supplemental material). Complementary $17-20$ bp oligonucleotide probes representative for each identified 6 bp sequence were selected in the context of the MMP-2 gene promoter (Fig. 3A) (supplemental Table 6, available at www.jneurosci.org as supplemental material) and tested for binding to recombinant His-tagged AT3Q23 and AT3Q70 in EMSAs.

AT3Q23 bound most intensely and apparently stronger than
AT3Q70 to probes containing the sequence motif (G/C)AGAAG (A3/4, A6-1) (Fig. 3B) and generated a specific band shift that was reduced by the addition of an AT3-specific antibody or the respective unlabeled oligonucleotide. This shift was not generated by recombinant His-tagged HDAC3 (Fig. 3D). Probes containing variations of this motif were bound by neither AT3Q23 nor AT3Q70 (A1-1, A5-2, A10-2) (Fig. 3B). In contrast, probes containing the repetitive sequence AGGAGG(A/T) (A2-2, A4-2, A5-1) were bound by both AT3Q23 and AT3Q70 (Fig. 3C) and formed a specific high molecular band shift that was prevented by the AT3 antibody or the respective unlabeled oligonucleotide and was not observed with HDAC3 (Fig. 3E). AT3Q70 produced a more intense band shift with probe A5-1 containing the sequence TAGGAGGAA, whereas AT3Q23 generated a stronger shift with probe A4-2 containing the sequence CAGGAGGAG (Fig. $3 C, E, G)$. Probes containing nucleotide substitutions at the be- 


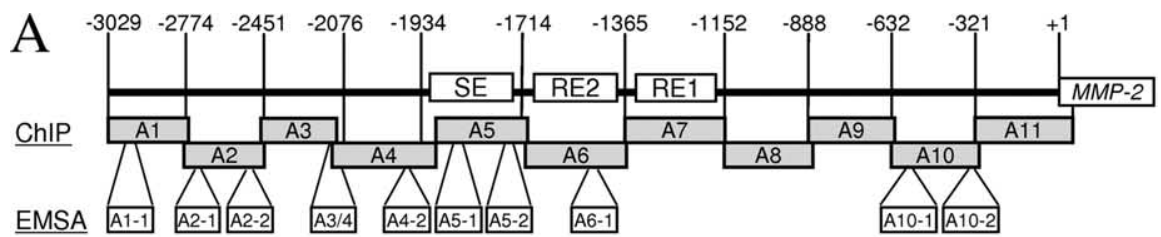

B
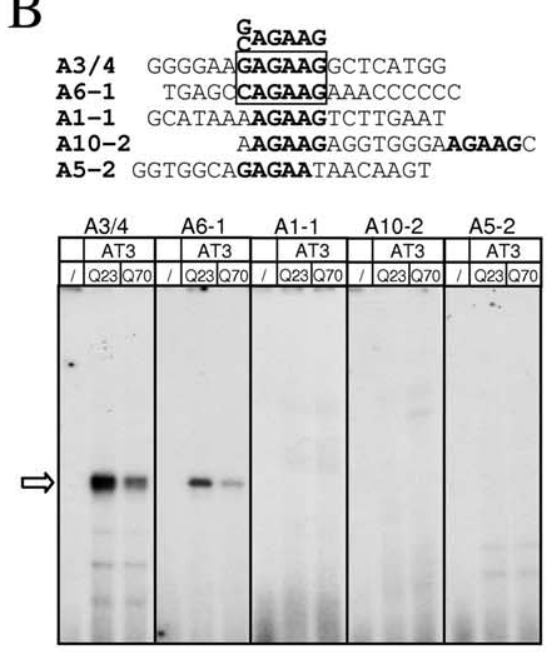

$\mathrm{D}^{\text {A3/4 }}$
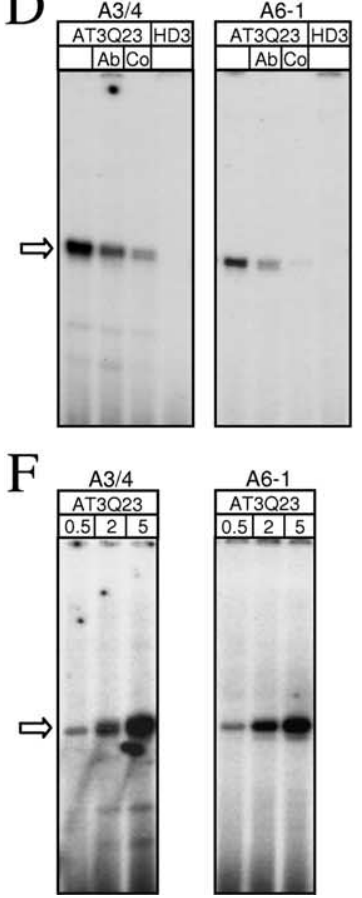

C
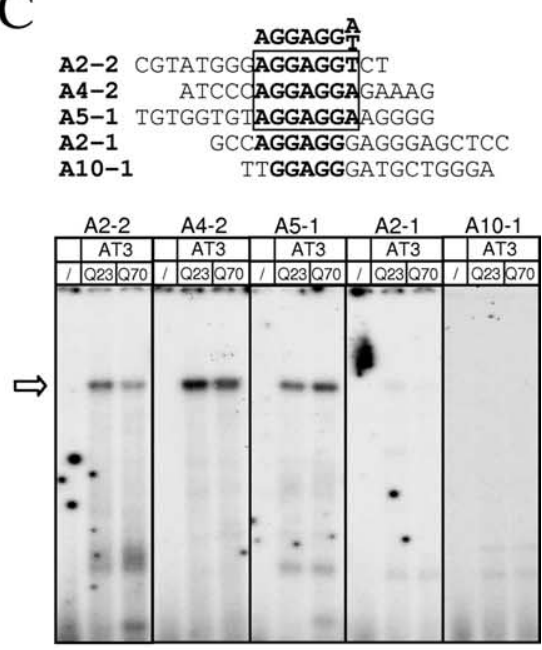

$\mathrm{E}$
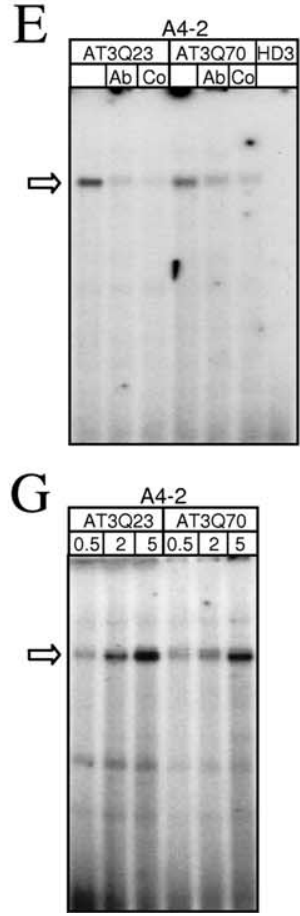

ginning or end of the repetitive sequence were not bound by AT3Q23 or AT3Q70 (A2-1, A10-1) (Fig. 3C). The protein amounts of AT3Q23 or AT3Q70 required to produce detectable shifts with the AGGAGGA-containing probes (A4-2, A5-1) (Fig. 3G) were approximately four times higher than the amount of AT3Q23 required to generate shifts with the $(\mathrm{G} /$ C)AGAAG-containing probes (A3/4, A6-1) (Fig. 3F). The selective binding of AT3Q23 and AT3Q70 to specific DNA sequences located in distinct regions of the MMP-2 promoter corresponded to a great extent to the AT3-enriched promoter regions in ChIP assays of Q23 (A4 and A6) and Q70 cells (A5) (Fig. 2D), suggesting that the different binding properties of each AT3 isoform may have caused the differential chromatin binding.

Among the chromatin clones so far analyzed in the library, one contained a genomic fragment of the interleukin-6 (IL-6) gene. Because IL-6 is upregulated in SCA3 cells and human brains (Evert et al., 2003), IL-6 may represent another potential target gene regulated by AT3. Other identified genes of the AT3-bound chromatin library encode, for instance, a kallikrein-binding protein, a steroid dehydrogenase, a cysteine-rich glycoprotein, Hsp70, and several receptors such as the interleukin-21 or cadherin-related receptor (supplemental Table 5, available at www. jneurosci.org as supplemental material).

AT3 interacts with HDAC3 and NCoR in SCA3 cell lines and human brain tissue

The correlation of AT3-bound with HDAC3- and NCoR-bound promoter regions suggested that AT3 associates with HDAC 3 and NCoR for transcriptional repression of the MMP-2 gene promoter. To analyze whether AT3 interacts with HDAC 3 and NCoR, we performed coimmunoprecipitations and GST fusion protein pulldown experiments by using

Figure 3. AT3 binds to specific DNA sequences of the MMP-2 promoter. A, Location of selected oligonucleotide probes for EMSA in relation to the amplified ChIP regions across the MMP-2 gene promoter. The selected MMP-2 probes $\left(5^{\prime}-3^{\prime}\right)$ contain potential AT3 regulatory 6 bp sequences identified in subcloned AT3-bound chromatin fragments (see Materials and Methods). $B$, EMSAs and oligonucleotide sequences of MMP-2 probes used for the identification of the sequence motif (G/C)AGAAG (box; identical nucleotides of the motif are shown in bold). The double-stranded labeled probes were incubated with no protein (/) or His-tagged AT3Q23 or AT3Q70 (each $5 \mu \mathrm{g}$ ). AT3Q23 produced a stronger shift than AT3Q70 with probes (A3/4 and A6-1) containing the sequence motif (G/C)AGAAG, whereas band shifts with probes containing variations of this motif(A1-1, A10-2, and A5-2) were not generated. C, EMSAs and oligonucleotide sequences of MMP-2 probes used for the identification of the sequence motif AGGAGG(A/T) (box; identical nucleotides of the motif are shown in bold). Both His-tagged AT3Q23 and His-tagged AT3Q70 (each 5 $\mu \mathrm{g}$ ) produced a high molecular band shift with probes (A2-2, A4-2, and A5-1) containing the repetitive sequence AGGAGG(A/T), whereas alterations of this sequence motif in probes (A2-1 and A10-1) did not generate band shifts. $D$, EMSAs confirming the specificity of the AT3Q23 band shifts obtained with the probes A3/4 and A6-1. The addition of an anti-AT3 antibody (Ab) or an excess of the respective unlabeled competing oligonucleotide probes $\left(C_{0}\right)$ strongly reduced formation of the band shifts obtained with His-AT3Q23 (5 $\mu \mathrm{g}$ ). Incubation of His-tagged HDAC3 (HD3; $5 \mu \mathrm{g}$ ) with the labeled probes A3/4 and A6-1 did not produce band shifts. E, EMSAs confirming the specificity of the AT3Q23 and AT3070 band shifts obtained with the probes A4-2 and A5-1.

The addition of the AT3 antibody or the respective unlabeled oligonucleotide probes prevented formation of the band shifts obtained for A4-2 and A5-1 with His-AT3Q23 or HisAT3Q70 (each $5 \mu \mathrm{g}$ ). His-HDAC3 $(5 \mu \mathrm{g})$ bound neither of the probes. $F$, DNA binding studies for probes $A 3 / 4$ and A6-1, using $0.5,2.0$, and $5.0 \mu \mathrm{g}$ of protein of His-AT3023 in EMSAs. Probes were bound sufficiently by using $0.5 \mu \mathrm{g}$ of AT3Q23. G, DNA binding studies for A4-2 and A5-1, using $0.5,2.0$, and 5.0 $\mu \mathrm{g}$ of protein of His-AT3Q23 and His-AT3Q70 in EMSAs. AT3Q23 showed stronger binding to A4-2 than did AT3070, whereas AT3070 bound stronger to A5-1 as compared with AT3023. Gels were exposed to $x$-ray films overnight $(\boldsymbol{B}-\boldsymbol{E})$ or for $48 \mathrm{~h}(\boldsymbol{F}, \boldsymbol{G})$. The positions of the specific band shifts are indicated by arrows. 
A
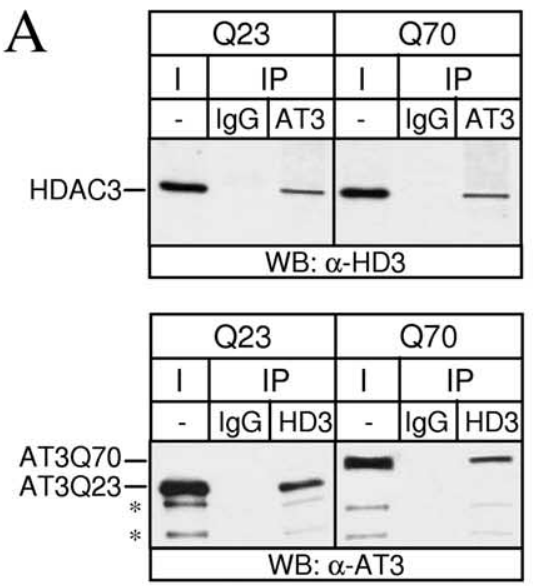

$\mathrm{C}$

\begin{tabular}{|l|c|c|c|c|}
\hline Ctrl & 1 & + & + & + \\
\hline GST & & + & & \\
\hline GST-AT3Q23 & & & + & \\
\hline GST-AT3Q70 & & & & + \\
\hline
\end{tabular}

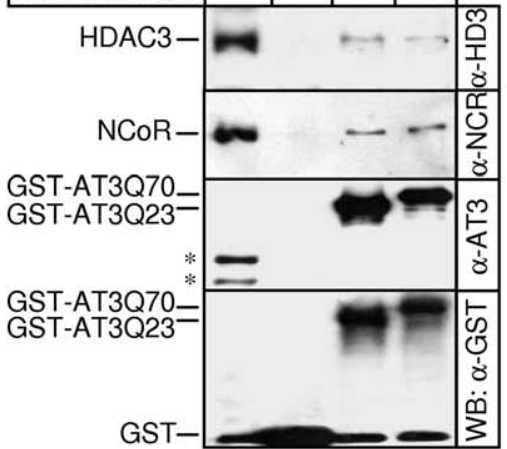

$\mathrm{E}$
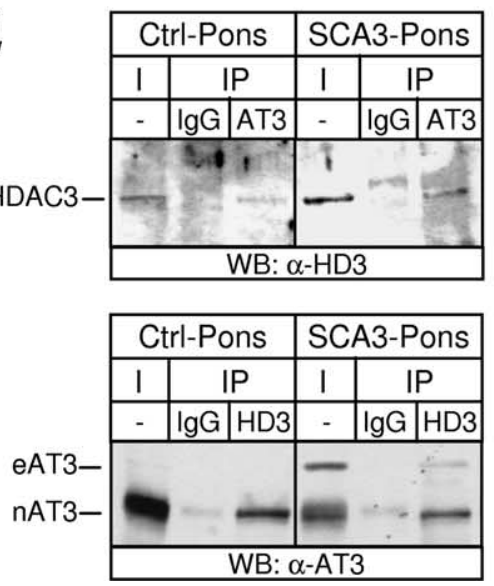

B
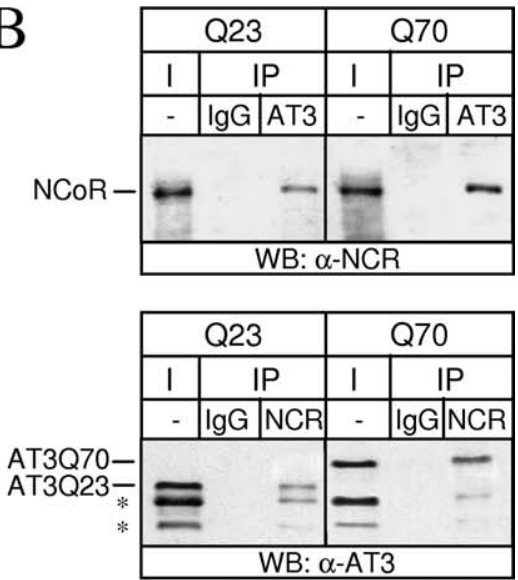

$\mathrm{D}$

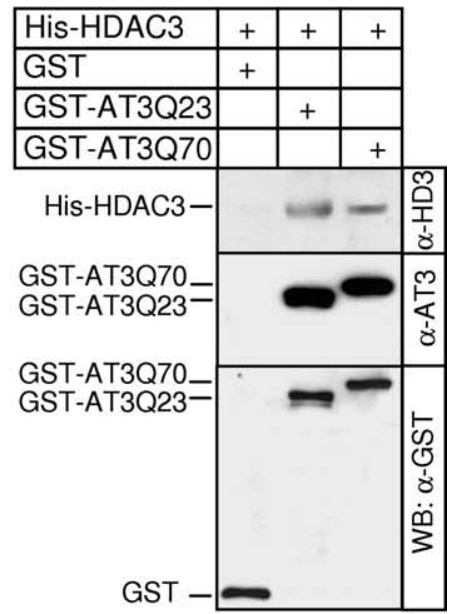

$\mathrm{F}$
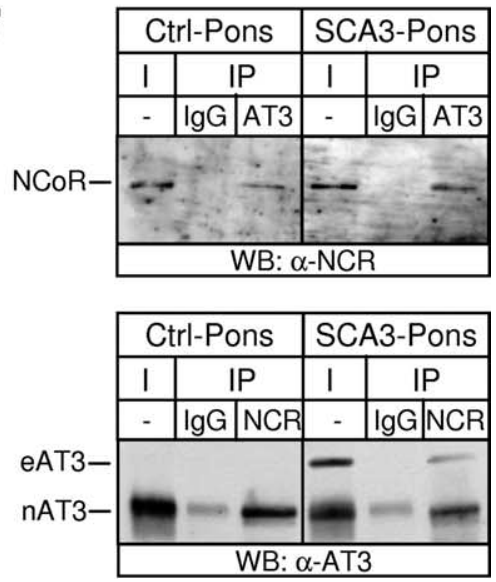

Figure 4. AT3 interacts with HDAC3 and NCOR. $A$, Western blot (WB) analysis of HDAC3 (top) and AT3 (bottom), using immunoprecipitated (IP) complexes from nuclear extracts of SCA3 cell lines (Q23 and Q70) with anti-AT3 (AT3), anti-HDAC3 (HD3), and control lgG (lgG) antibodies. $B$, WB analysis of NCoR (top) and AT3 (bottom), using immunoprecipitated complexes from nuclear extracts of SCA3 cell lines (Q23 and Q70) with anti-AT3, anti-NCOR (NCR), and control lgG antibodies. $\boldsymbol{A}$ and $\boldsymbol{B}$ show that both endogenous HDAC3 and NCOR interact with normal and expanded AT3 as well as with endogenous rat AT3 isoforms; asterisks indicate endogenous AT3 isoforms. Input (I) represents $10 \%$ of the protein amount used for each IP. C, Pulldown experiments using nuclear extracts of control (Ctrl) cells incubated with purified GST, GST-AT3Q23, and GST-AT3070 fusion proteins immobilized on glutathione-Sepharose beads. Beads were washed, and bound proteins were eluted and analyzed by WB, using antibodies against $\mathrm{HDAC} 3, \mathrm{NCOR}$, AT3, and GST. Endogenous $\mathrm{HDAC} 3$ and NCOR were enriched selectively from the nuclear Ctrl extract with the fusion proteins GST-AT3Q23 and GST-AT3070, but not with GST alone. Input represents 10\% of the protein amount used for each pulldown; asterisks indicate endogenous AT3 isoforms. D, In vitro binding assay of GST, GST-AT3Q23, and GST-AT3Q70 fusion proteins immobilized on glutathione-Sepharose beads incubated with purified His-tagged HDAC3. Beads were washed, and bound proteins were eluted and analyzed by WB, using antibodies against HDAC3, AT3, and GST. Pulldown of GST-AT3Q23 and GST-AT3Q70 showed that both AT3 isoforms are capable of interacting directly with His-HDAC3.E, WB analysis of HDAC3 (top) and AT3 (bottom), using immunoprecipitated complexes from human pons extracts of an unaffected control

nuclear extracts of SCA3 cell lines. Immunoprecipitations with an AT3-specific antibody showed that both endogenous HDAC3 (Fig. 4A, top) and NCoR (Fig. $4 B$, top) coprecipitated with AT3 in Q23 and Q70 cells. Correspondingly, immunoprecipitations with an HDAC3- and NCoR-specific antibody revealed that both normal and expanded AT3 as well as the endogenous rat isoforms of AT3 coprecipitated with $\mathrm{HDAC} 3$ and NCoR (Fig. $4 A, B$, bottom). To confirm the coimmunoprecipitation studies, we purified and incubated GST fusion proteins of AT3Q23 and AT3Q70 with nuclear extracts of Ctrl cells. After the addition and pulldown of GST beads, endogenous HDAC3 and NCoR were enriched selectively from cell extracts with the fusion proteins GSTAT3Q23 and GST-AT3Q70 (Fig. 4C). Moreover, in vitro binding assays that used purified His-tagged HDAC3 with GSTAT3Q23 and GST-AT3Q70 confirmed that both AT3 isoforms were capable of interacting directly with HDAC3 (Fig. 4D). GST-AT3Q23 enriched His-HDAC3 more efficiently than GST-AT3Q70. However, the levels of precipitated endogenous HDAC3 in nuclear extracts of the SCA3 cell lines were comparable, indicating that the interaction was not affected by the polyQ repeat length. To verify these findings in human brain, we prepared extracts from frozen pons tissue of controls and SCA3 patients. With the AT3 antibody both endogenous HDAC3 (Fig. $4 E$, top) and NCoR (Fig. 4F, top) coprecipitated with AT3 from both the control and SCA3 patient. Reciprocally, the endogenous non-expanded (nAT3) and expanded (eAT3) AT3 isoforms from human pons extracts coprecipitated with $\mathrm{HDAC} 3$ and NCoR (Fig. 4E, F, bottom). A minor reduction of coimmunoprecipitated, expanded AT3 as compared with the non-expanded AT3 isoform (Fig. 4E, F, bottom) was apparent in SCA3 pons. However, the levels of coprecipitated HDAC3 and NCoR in human brain extracts and SCA3 cell lines were not strikingly different, confirming that the protein-protein interaction was not affected by the polyQ expansion in AT3. Coimmunofluorescence staining of

$\leftarrow$

and an SCA3 patient with antibodies against AT3, HDAC3, and control lgG. $F$, WB analysis of NCOR (top) and AT3 (bottom), using immunoprecipitated complexes from a Ctrl and SCA3 human pons extract with antibodies against AT3 and NCOR. E and $\boldsymbol{F}$ show that both endogenous HDAC3 and NCOR physiologically interact with endogenous non-expanded (nAT3) and expanded AT3 (eAT3) in extracts from human pons tissue. Input represents $10 \%$ of the protein amount used for each IP. 


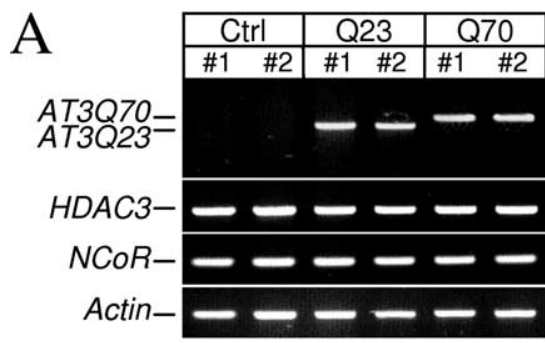

$\mathrm{C}$
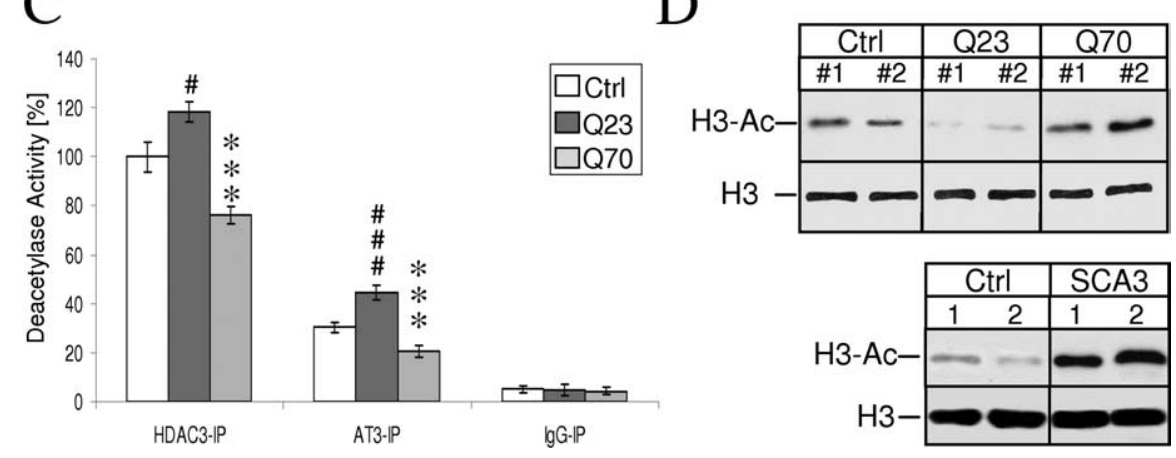

Figure 5. AT3 alters HDAC activity and histone $H 3$ acetylation status. $\boldsymbol{A}$, Semiquantitative $P C R$ analysis for endogenous $H D A C 3$, NCOR, and actin expression as well as recombinant human normal (AT3Q23) and expanded (AT3Q70) AT3 expression, using CDNA prepared from individual clonal (\#1 and \#2) SCA3 cell lines (Ctrl, Q23, and Q70). B, Western blot analysis of AT3, HDAC3, NCOR, and actin in nuclear extracts prepared from individual clonal (\#1 and \#2) SCA3 cell lines (Ctrl, Q23, and Q70). Recombinant human normal (AT3Q23) and expanded AT3 (AT3Q70) isoforms are indicated; asterisks indicate endogenous rat AT3 isoforms. $\boldsymbol{A}$ and $\boldsymbol{B}$ show that HDAC3 and NCOR were expressed at comparable levels both at the mRNA and nuclear protein levels in individual clonal SCA3 cell lines. C, HDAC activities of immunoprecipitated complexes from nuclear extracts of SCA3 cell lines (Ctrl, Q23, and Q70) with antibodies against AT3, HDAC3, or control lgG. Immunoprecipitations of both AT3-and HDAC3-containing protein complexes from Q70 cells showed significantly decreased deacetylase activities as compared with Ctrl and Q23 cells ( ${ }^{* * *} p<0.001$; ANOVA followed by Tukey-Kramer). Deacetylase activities of immunoprecipitated complexes from 023 cell lines were increased significantly as compared with Ctrl cells ( ${ }^{\#} p<0.05$; \#\# $p<0.001$; ANOVA, followed by Tukey-Kramer). Each of the presented groups contains the results obtained from two individual clonal cell lines (\#1 and \#2) from each stable SCA3 cell line (Ctrl, Q23, and Q70) and represent the mean $\pm S D(n=6)$. HDAC activities are displayed as a percentage of fluorescence units emitted from HDAC3precipitated complexes in Ctrl cells. D, Acetylation of histone $\mathrm{H} 3$ was determined by Western blot analysis of histones extracted from individual clonal (\#1 and \#2) SCA3 cell lines (Ctrl, Q23, and Q70; top) and from human pons tissue (bottom), using a specific antibody for acetylated histone $\mathrm{H3}(\mathrm{H3}-\mathrm{Ac}$ ). Histones extracted from $\mathrm{Q} 23$ clonal cell lines showed reduced levels of acetylated histone H3, whereas Ctrl and Q70 clonal cell lines had normal or increased levels, respectively, of acetylated histone $\mathrm{H} 3$ each as compared with the total amount of immunoreactive $\mathrm{H} 3$. Human pons tissue showed increased levels of acetylated histone $\mathrm{H} 3$ in SCA3 patients as compared with the levels of acetylated histone $\mathrm{H} 3$ in controls. Total histone $\mathrm{H} 3$ protein was detected in parallel blots with anti-histone $\mathrm{H} 3$ antibody.

human pons sections from controls and SCA3 patients additionally showed that neither HDAC3 nor NCoR was recruited into NIs formed by AT3 (supplemental Fig. $1 A, B$, available at www.jneurosci.org as supplemental material). These findings show that HDAC3 and NCoR physiologically interact with AT3 independently of the polyQ repeat length and without being recruited into NIs.

\section{AT3 alters HDAC activity and histone acetylation}

HDAC3 3 and NCoR were expressed at comparable levels both at the mRNA (Fig. 5A) and nuclear protein level (Fig. $5 B$ ) in individual clonal SCA3 cell lines and human brain (Fig. $4 E, F$ ) (supplemental Fig. $1 A, B$, available at www.jneurosci.org as supplemental material). Thus neither altered gene expression nor altered protein abundance of $\mathrm{HDAC} 3$ and $\mathrm{NCoR}$ accounts for the differential expression of MMP-2. Because AT3 inhibits proteasomal degradation of polyubiquitylated model substrates (Burnett and Pittman, 2005) and HDAC3 is degraded by the UPS (Zhang et al., 1998; Perissi et al., 2004; Dennis et al., 2005), we analyzed whether the interaction of AT3 with HDAC3 alters the ubiquitylation/deubiquitylation status of HDAC3 or influences
HDAC3 deacetylase activity. Immunoprecipitations of endogenous HDAC3 from nuclear extracts of the SCA3 cell lines and immunoblotting with two antibodies against monoubiquitin and polyubiquitin chain conjugates did not reveal differentially ubiquitylated HDAC3 isoforms (data not shown). However, immunoprecipitated AT3- and HDAC3-containing protein complexes from Q70 cell lines showed significantly decreased deacetylase activities as compared with $\mathrm{Ctrl}$ and Q23 cells (Fig. 5C). Moreover, both the AT3- and HDAC3-associated deacetylase activities of Q23 cells were significantly higher than in Ctrl cells (Fig. 5C), showing that normal AT3 is associated with increased deacetylase activities and expanded AT3 is associated with reduced deacetylase activities of HDACcontaining protein complexes. The changes of the deacetylase activities were reflected by an altered acetylation status of histone H3. Compared with the total amount of immunoreactive $\mathrm{H} 3$ protein, Q23 cell lines showed reduced levels of acetylated histone $\mathrm{H} 3$, whereas $\mathrm{Ctrl}$ and Q70 cell lines had normal or increased levels, respectively, of acetylated histone $\mathrm{H} 3$ (Fig. 5D, top). Thus normal AT3 is associated with increased deacetylase activity of HDAC3-containing protein complexes and increased deacetylation of histone $\mathrm{H} 3$, whereas expanded AT3 correlates with reduced deacetylase activity and increased histone $\mathrm{H} 3$ acetylation. Consistent with these findings, histone $\mathrm{H} 3$ extracted from frozen human pons tissue showed increased levels of acetylated histone $\mathrm{H} 3$ in two SCA3 patients as compared with the levels of acetylated histone $\mathrm{H} 3$ in two controls (Fig. $5 D$, bottom).

\section{AT3 and HDAC3 repress MMP-2 gene transcription}

To analyze whether AT3 functionally cooperates with HDAC3 in transcriptional repression of the MMP-2 gene, we measured luciferase activities of native CSM14.1 cells transiently transfected with HDAC3 or normal (AT3Q23) or expanded AT3 (AT3Q70) (Fig. $6 \mathrm{~B}$ ) along with the full-length MMP-2 reporter construct (T1) (Fig. 6A). Consistent with our findings in the stable SCA3 cell lines, transient expression of AT3Q23 repressed MMP-2 transcription by $\sim 40 \%$, whereas AT3Q70 increased it by $\sim 60 \%$ each when compared with basal levels of MMP-2 transcription in empty vector-transfected CSM14.1 cells (Fig. 6A). Transient expression of HDAC3 alone reduced the MMP-2 promoter activity by $\sim 40 \%$ (Fig. $6 \mathrm{~A}$ ). The repressive effect was enhanced when normal AT3 was coexpressed along with HDAC3 (Fig. 6A). Coexpression of HDAC3 strongly repressed the aberrant transcriptional activation of expanded AT3 and reduced luciferase activity to basal levels (Fig. 6A). Consistent with these findings, transient expression of AT3Q23 or AT3Q70 in native CSM14.1 cells resulted in repression or activation, respectively, of the endogenous MMP-2 gene expression, both at the level of MMP-2 mRNA (Fig. 

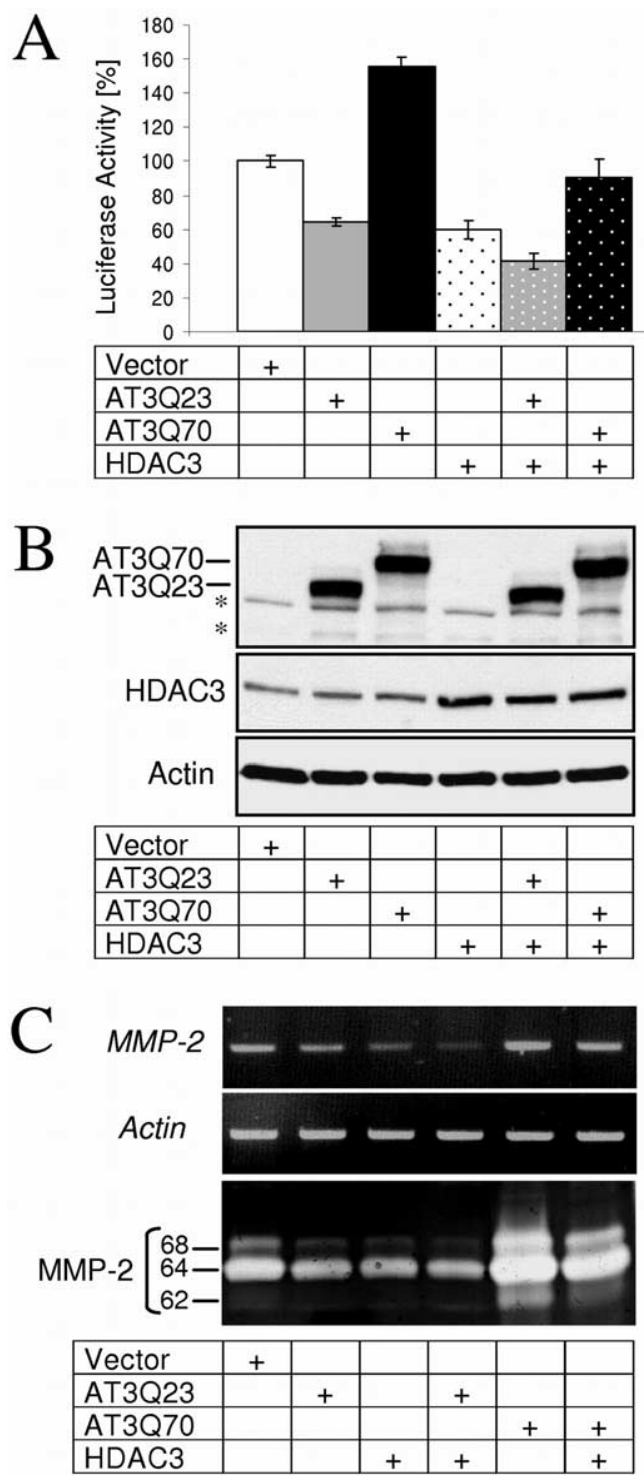

Figure 6. AT3 and HDAC3 mediate transcriptional repression of the MMP-2 gene expression. $\boldsymbol{A}$, Reporter assays of transiently transfected native CSM14.1 cells using the MMP-2 full-length promoter construct T1 along with the expression constructs encoding normal (AT3Q23), expanded AT3 (AT3Q70), or HDAC3. AT3Q23 repressed and AT3Q70 increased the transcriptional activation of the MMP-2 promoter construct. HDAC3 enhanced the normal AT3-mediated repression and prevented the aberrant activation of MMP-2 by expanded AT3. The luciferase activities are presented as a percentage of activity measured in empty vector ( $p c D N A 3$ ) transfected cells along with the full-length promoter construct T1. The results were averaged from four independent experiments $(n=4)$ and are presented as the mean $\pm S D$. $\boldsymbol{B}$, Western blot analysis of the transiently transfected CSM14.1 cells in $\boldsymbol{A}$ showing that all constructs were expressed appropriately. Protein (50 $\mu \mathrm{g})$ of the reporter cell lysates was used for immunodetection with antibodies against AT3, HDAC3, and actin; asterisks indicate endogenous rat AT3 isoforms. C, Semiquantitative $P C R$ analysis for endogenous MMP-2 and actin expression, using CDNA (top) from native CSM14.1 cells transiently transfected with constructs encoding AT3Q23, AT3Q70, or HDAC3. Shown is gelatin zymography of secreted, catalytically active MMP-2 isoforms using simultaneously isolated media of transiently transfected native CSM14.1 cells (bottom). Cleared proteolytic zones indicate the presence of gelatinases at their respective molecular weights and are assigned to the latent form of pro-MMP-2 $(68 \mathrm{kDa})$ and the intermediate and fully activated forms of MMP-2 (64 and $62 \mathrm{kDa}$, respectively). Transient expression of AT3Q23 or AT3Q70 in native CSM14.1 cells resulted in repression or activation, respectively, of the endogenous MMP-2 gene expression, both at the level of MMP-2 mRNA and the level of secreted proteolytically active MMP-2; coexpression/expression of HDAC3 repressed MMP-2 expression.
$6 C$, top) and the level of secreted proteolytically active MMP-2 (Fig. $6 C$, bottom). Accordingly, coexpression/expression of HDAC3 repressed MMP-2 expression. These findings show that normal AT3 and HDAC3 mediate transcriptional repression, whereas expanded AT3 aberrantly activates transcription of the MMP-2 gene.

\section{Properties of AT3 required for transcriptional repression or activation}

To address whether the transcriptional regulatory property of AT3 requires the catalytic cysteine $(\mathrm{C} 14)$ of its protease domain or the conserved leucine residues (L229 and L249) in its UIMs (Fig. 7A), we analyzed the effects of active site (C14A) and/or UIM (L229/ 249A) mutants of both normal (AT3Q23) and expanded AT3 (AT3Q70) on MMP-2 transcription in reporter assays (Fig. 7B), using the MMP-2 promoter construct T1 and native CSM14.1 cells. The active site mutant of normal AT3 (AT3Q23-C14A) resulted in a weak loss of its repressing activity, whereas the UIM mutant (AT3Q23-L229/249A) relieved repression and instead enhanced MMP-2 transcription (Fig. $7 B$ ). Normal AT3 with both the active site and UIMs mutated (AT3Q23-C14A-L229/249A) neither activated nor repressed MMP-2 transcription (Fig. $7 B$ ). In contrast, the active site mutant of expanded AT3 (AT3Q70-C14A) almost entirely abolished the aberrant transcriptional activation capacity of expanded AT3, whereas the UIM mutations (AT3Q70-L229/249A) did not influence the enhanced transcriptional activation of MMP-2 (Fig. 7B). Expanded AT3 with both the active site and UIMs mutated (AT3Q70-C14A-L229/249A) lost its transactivating capacity (Fig. $7 B$ ). These findings also were confirmed at the level of secreted, proteolytically active MMP-2 isoforms (Fig. 7C). Transient expression of AT3Q70-C14A resulted in decreased expression and AT3Q23-L229/249A in increased expression, respectively, of proteolytically active MMP-2 (Fig. 7C). Thus the catalytic cysteine residue of expanded AT3 is associated with the aberrant transcriptional activation of the MMP-2 gene, whereas the UIMs of normal AT3 are essential for the transcriptional repression of the MMP-2 gene transcription.

\section{Discussion}

In the present study we provide evidence that AT3 binds to specific sites in chromatin regions of the MMP-2 gene promoter and interacts with the transcriptional corepressors HDAC3 and NCoR, resulting in deacetylation of histones and reduced binding of the transcription factor GATA-2 to target regions of the MMP-2 promoter. Expanded AT3 showed altered DNA and chromatin binding, failed to accomplish functional repressor complexes on the promoter, and aberrantly activated MMP-2 expression via increased histone acetylation and GATA-2 binding. Normal and expanded AT3 interacted with endogenous HDAC3 and NCoR in cell and human brain extracts, but only normal AT3 was associated with increased deacetylase activities and deacetylation of histone $\mathrm{H} 3$, indicating that AT3 represses gene transcription via histone deacetylation.

Previously, AT3 had been shown to bind histone proteins and to repress the coactivator-mediated transcription in vitro (Chai et al., 2001; Li et al., 2002). Li and coworkers proposed that AT3 may function as an inhibitor of histone acetyltransferases (INHAT) subunit and inhibits transcription via histone masking and blocking access of HATs to acetylation sites on histones. Subunits of INHAT complexes typically repress transcription via the recruitment of HDACs and NCoR and deacetylation of histone H3 (Seo et al., 2001; Kutney et al., 2004; Schneider et al., 2004; Macfarlan et al., 2005). HDACs function within active multiprotein complexes directed to the promoters of repressed genes. HDAC3 is a component 


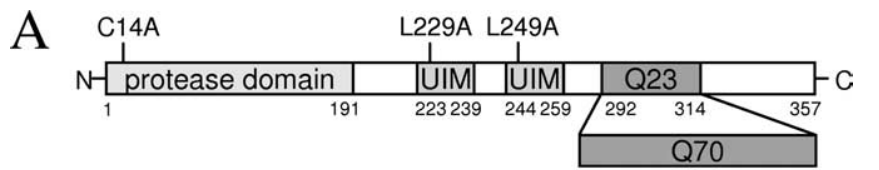

B

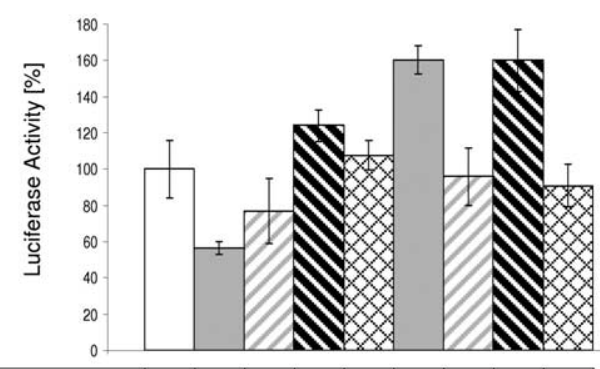

\begin{tabular}{|l|l|l|l|l|l|l|l|l|l|}
\hline Vector & + & & & & & & & & \\
\hline AT3Q23 & & + & & & & & & & \\
\hline AT3Q23-C14A & & & + & & & & & & \\
\hline AT3Q23-L229/249A & & & & + & & & & & \\
\hline AT3Q23-C14A-L229/249A & & & & & + & & & & \\
\hline AT3Q70 & & & & & & + & & & \\
\hline AT3Q70-C14A & & & & & & & + & & \\
\hline AT3Q70-L229/249A & & & & & & & & + & \\
\hline AT3Q70-C14A-L229/249A & & & & & & & & & + \\
\hline
\end{tabular}

C

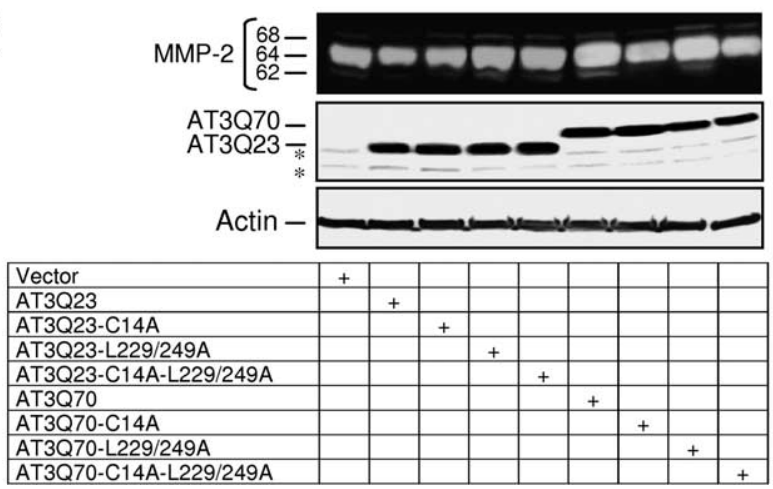

Figure 7. AT3 requires its ubiquitin-associated functions to repress MMP-2 gene transcription. $A$, Schematic of full-length human AT3 expression constructs with normal (Q23) and expanded poly $Q$ repeat (Q70). The mutated positions of the ubiquitin protease domain (C14A) and UIMs (L229A and L249A) are indicated. B, Reporter assays of transiently transfected native CSM14.1 cells using the promoter construct $\mathrm{T} 1$ along with the expression constructs encoding the AT3 mutants C14A, L229/249A, and C14A-L229/249A of normal (AT3023) and expanded AT3 (AT3Q70). The UIM mutant of normal AT3 (AT3Q23-L229/249A) relieved repression and instead enhanced MMP-2 transcription. In contrast, the active site mutant of expanded AT3 (AT3070-C14A) almost entirely abolished the aberrant transcriptional activation capacity of expanded AT3. The luciferase activities are presented as a percentage of the activity measured in empty vector ( $p(D N A 3$ ) transfected cells along with the full-length promoter construct T1. The results were averaged from four independent experiments $(n=4)$ and are presented as the mean \pm SD. C, Gelatin zymography (top) of secreted, catalytically active MMP-2 forms using concentrated media of the transiently transfected native CSM14.1 in B confirmed that transient expression of AT3Q70-C14A results in decreased expression and AT3023-L229/249A in increased expression, respectively, of proteolytically active MMP-2. Western blot analysis (bottom) of the transiently transfected CSM14.1 cells confirms appropriate expression of all AT3 constructs. Protein $(50 \mu \mathrm{g})$ of the reporter cell lysates in $\boldsymbol{B}$ was used for immunodetection with antibodies against AT3 and actin; asterisks indicate endogenous rat AT3 isoforms. Reporter cell lysates and concentrated medium were prepared simultaneously from the transfected cells.

of a large protein complex containing NCoR, the NCoR-related corepressor SMRT, TBL1 (transducin $\beta$-like protein 1) and TBLR1 (TBL1-linked receptor 1) (two highly related WD-40 repeat proteins), and GPS2 (G-protein pathway suppressor 2), a cellular signaling protein (Li et al., 2000; Guenther et al., 2001; Zhang et al., 2002; Yoon et al., 2003). The interaction and colocalization of normal AT3 with HDAC3 and NCoR in transcriptionally repressed, histone-deacetylated chromatin regions of the MMP-2 promoter suggest that AT3 mediates repression via the
NCoR/SMRT multiprotein repressor complex. We previously found pp32/LANP, a major component of INHAT complexes (Seo et al., 2001, 2002), in NIs formed by expanded AT3 in affected neurons of SCA3 patients (Evert et al., 2003). Similar data also have been obtained in SCA1; expanded ataxin-1 (AT1) interacts with pp32/LANP and alters its subnuclear localization (Matilla et al., 1997). Thus AT3 and probably AT1 may function as INHAT components via chromatin binding, recruitment of HDACs and $\mathrm{NCoR}$, histone deacetylation, and repression of specific target genes.

Recently, it has been shown that AT1 interacts with HDAC3 and SMRT independently of the glutamine repeat length to repress transcription. In addition, expanded AT1 colocalizes with SMRT to the same chromosomal regions in a Drosophila model (Tsai et al., 2004). We found that only normal AT3 colocalized with HDAC3 and NCoR in chromatin regions of the MMP-2 gene promoter that also were repressed transcriptionally in reporter assays. Expanded AT3 bound to distinctively separate chromatin regions and no longer was associated with recruitment of HDAC3, NCoR, HDAC, and transcriptional repression of the MMP-2 promoter. Moreover, expanded AT3 showed altered binding to specific DNA sequences located in transcriptionally repressed promoter regions. It is thus likely that the polyQ expansion in AT3 changes its DNA binding properties and causes altered chromatin binding not associated with corepressor recruitment. The identified DNA binding sequences for AT3 represent hitherto unknown transcription factor motifs but resemble the binding site (GAGGAA) of E-twenty-six domain (ETS) family members (Scott et al., 1994). Interestingly, three of the five AT3-bound MMP-2 probes (A3/4, A4-2, and A5-1) (Fig. 3) contain predicted binding sites of ETS transcription factors according to the TRANSFAC database (database on transcription factors). AT3 does not possess a winged helix-turn-helix DNA binding domain (Albrecht et al., 2003; Scheel et al., 2003) of ETS factors but contains a basic leucine zipper (bZIP) region (223-270 aa) according to the SMART (Simple Modular Architecture Research Tool) database. bZIP proteins bind to DNA via a leucine zipper structure that is required for dimerization and an adjacent basic region that directly contacts DNA (Landschulz et al., 1988). ETS and bZIP factors are important for the regulation of cytokine genes such as IL-1 $\beta$ (Yang et al., 2000) and IL-6 (Akira et al., 1990; Nishiyama et al., 2004). Indeed, we identified an AT3-bound genomic fragment of the IL- 6 gene and previously have found upregulation of IL-6 in SCA3 cells and human brains (Evert et al., 2003), indicating that IL-6 may represent another potential target gene of AT3. Future studies are required to analyze larger sets of AT3-bound genomic regions and the expression of the corresponding genes to generate a comprehensive list of AT3-regulated genes.

Expanded AT3 increased MMP-2 transcription and was associated with decreased deacetylase activities and increased acetylation of histone $\mathrm{H} 3$, suggesting that failure of expanded AT3 to accomplish functional repressor complexes on target gene promoters increases gene transcription. The molecular mechanism involves not only diminished HDAC but additional cis-regulatory elements residing in the target gene promoter. The RE2 enhancer region of the MMP-2 gene contains GATA-2 binding sites and can be activated by the expression of GATA-2 (Han et al., 2003). We showed that transcriptional activation of MMP-2 was repressed when GATA-2 binding sites were mutated in RE2. Moreover, GATA-2 was enriched strongly within RE2 and two additional regions of the MMP-2 pro- 
moter, mediating transcriptional activation in Ctrl and Q70 cells. Thus the transcriptional activation of MMP-2 in Ctrl and Q70 cells is mediated by GATA-2. The interaction between HDAC3 and GATA-2 is proposed to result in deacetylation and decreased binding affinity of GATA-2 (Ozawa et al., 2001), whereas the DNA binding affinity of GATA-2 increases via acetylation by HATs (Hayakawa et al., 2004). Accordingly, Q23 cells show minimal recruitment of GATA-2 to its target promoter regions but reveal strong recruitment of HDAC3. It remains an open question whether AT3 in addition to its effects on histone acetylation also interferes with the acetylation of specific transcription factors such as GATA-2.

AT3 is a deubiquitylating cysteine protease and binds polyubiquitin chains via its UIMs (Burnett et al., 2003; Donaldson et al., 2003; Chai et al., 2004). In the absence of the catalytic cysteine residue the UIMs of AT3 inhibit degradation by the proteasome, resulting in accumulation of polyubiquitylated proteins (Berke et al., 2005; Burnett and Pittman, 2005). In the absence of functional UIMs the binding of polyubiquitin is abolished, but nonexpanded AT3 still removes ubiquitin chains. The active site and UIM mutants of AT3 in our experiments show that the UIMs are essential for the repressive transcriptional function of normal AT3. In contrast, the active site cysteine alone accounts for the aberrant transcriptional activation by expanded AT3, suggesting that expanded AT3 may have lost its UIMdependent, repressive function. This is consistent with a model of action in which the UIM region of AT3 is necessary to recognize ubiquitylated substrates and to regulate the N-terminal ubiquitin protease activity (Mao et al., 2005; Nicastro et al., 2005). These proteasome-associated functions fit well into the hypothesis that the AT3 transcriptional regulatory role involves deubiquitylation and increased stabilization of certain proteins required for transcriptional repression of specific genes such as MMP-2. A growing body of evidence indicates that the UPS influences transcription in several diverse ways, which range from the regulation of chromatin to the degradation of transcriptional activators (Hicke, 2001; Muratani and Tansey, 2003). Deubiquitylating enzymes are recruited actively during transcription to regulate the activity of specific subunits of the regulatory apparatus (Freiman and Tjian, 2003; Amerik and Hochstrasser, 2004). Thus the function of normal AT3 in transcription may be to regulate the proteolytic turnover of select components of a transcriptional repression complex on chromatin, illustrated in Figure 8.

Compared with the initial hypothesis that polyQ toxicity is determined by aggregation of the expanded disease proteins and formation of NIs, more complex and specific scenarios of gain and loss of function emerge for the different polyQ disorders. In Huntington's disease the interaction of huntingtin and Hip-1 is reduced by polyQ expansion, promoting the formation of a pro- apoptotic Hippi-Hip-1 heterodimers and recruitment of procaspase- 8 (Gervais et al., 2002). At the same time, huntingtin interacts with the transcriptional repressor element-1 transcription factor/neuron restrictive silencer factor to modulate the transcription of neuron restrictive silencer element-controlled genes (Zuccato et al., 2003). Third, huntingtin specifically enhances vesicular transport of brain-derived neurotrophic factor (BDNF) (Gauthier et al., 2004). Expanded AT3 not only loses its repressing function but acquires a new transcriptional activator function. The loss of repression is reflected by its altered chromatin binding and failure to recruit HDAC3 and NCoR and to deacetylate core histones of a target promoter. The new transcriptional activator function is mediated via its active site, which probably disturbs the formation or maintenance of histonedeacetylating repressor complexes on target promoters. Both the loss of repressor and gain of an activator function result in increased histone acetylation of specific target gene promoters, probably leading to altered gene expression in SCA3 (Evert et al., 2001, 2003). Moreover, because non-expanded AT3 is aggregated into NIs formed by expanded AT3 (Uchihara et al., 2001; Haacke et al., 2006), depletion of AT3 repressor activity in affected neurons in addition may promote the upregulation of physiologi- 
cally repressed genes. The identification of the array of genes directly regulated by AT3, such as MMP-2 and probably IL-6, will be important to clarify the pathophysiological events involved in SCA3.

\section{References}

Akira S, Isshiki H, Sugita T, Tanabe O, Kinoshita S, Nishio Y, Nakajima T, Hirano T, Kishimoto T (1990) A nuclear factor for IL-6 expression (NFIL6) is a member of a C/EBP family. EMBO J 9:1897-1906.

Albrecht M, Hoffmann D, Evert BO, Schmitt I, Wullner U, Lengauer T (2003) Structural modeling of ataxin-3 reveals distant homology to adaptins. Proteins 50:355-370.

Amerik AY, Hochstrasser M (2004) Mechanism and function of deubiquitinating enzymes. Biochim Biophys Acta 1695:189-207.

Berke SJ, Chai Y, Marrs GL, Wen H, Paulson HL (2005) Defining the role of ubiquitin-interacting motifs in the polyglutamine disease protein, ataxin-3. J Biol Chem 280:32026-32034.

Boutell JM, Thomas P, Neal JW, Weston VJ, Duce J, Harper PS, Jones AL (1999) Aberrant interactions of transcriptional repressor proteins with the Huntington's disease gene product, huntingtin. Hum Mol Genet 8:1647-1655.

Burnett B, Li F, Pittman RN (2003) The polyglutamine neurodegenerative protein ataxin- 3 binds polyubiquitylated proteins and has ubiquitin protease activity. Hum Mol Genet 12:3195-3205.

Burnett BG, Pittman RN (2005) The polyglutamine neurodegenerative protein ataxin-3 regulates aggresome formation. Proc Natl Acad Sci USA 102:4330-4335.

Chai Y, Wu L, Griffin JD, Paulson HL (2001) The role of protein composition in specifying nuclear inclusion formation in polyglutamine disease. J Biol Chem 276:44889-44897.

Chai Y, Berke SS, Cohen RE, Paulson HL (2004) Poly-ubiquitin binding by the polyglutamine disease protein ataxin-3 links its normal function to protein surveillance pathways. J Biol Chem 279:3605-3611.

Cho RJ, Campbell MJ, Winzeler EA, Steinmetz L, Conway A, Wodicka L, Wolfsberg TG, Gabrielian AE, Landsman D, Lockhart DJ, Davis RW (1998) A genome-wide transcriptional analysis of the mitotic cell cycle. Mol Cell 2:65-73.

de Chiara C, Menon RP, Dal Piaz F, Calder L, Pastore A (2005) Polyglutamine is not all: the functional role of the AXH domain in the ataxin-1 protein. J Mol Biol 354:883-893.

Dennis AP, Lonard DM, Nawaz Z, O'Malley BW (2005) Inhibition of the $26 \mathrm{~S}$ proteasome blocks progesterone receptor-dependent transcription through failed recruitment of RNA polymerase II. J Steroid Biochem Mol Biol 94:337-346.

Donaldson KM, Li W, Ching KA, Batalov S, Tsai CC, Joazeiro CA (2003) Ubiquitin-mediated sequestration of normal cellular proteins into polyglutamine aggregates. Proc Natl Acad Sci USA 100:8892-8897.

Doss-Pepe EW, Stenroos ES, Johnson WG, Madura K (2003) Ataxin-3 interactions with $\operatorname{Rad} 23$ and valosin-containing protein and its associations with ubiquitin chains and the proteasome are consistent with a role in ubiquitin-mediated proteolysis. Mol Cell Biol 23:6469-6483.

Evert BO, Wullner U, Schulz JB, Weller M, Groscurth P, Trottier Y, Brice A, Klockgether T (1999) High level expression of expanded full-length ataxin-3 in vitro causes cell death and formation of intranuclear inclusions in neuronal cells. Hum Mol Genet 8:1169-1176.

Evert BO, Vogt IR, Kindermann C, Ozimek L, de Vos RA, Brunt ER, Schmitt I, Klockgether T, Wullner U (2001) Inflammatory genes are upregulated in expanded ataxin-3-expressing cell lines and spinocerebellar ataxia type 3 brains. J Neurosci 21:5389-5396.

Evert BO, Vogt IR, Vieira-Saecker AM, Ozimek L, de Vos RA, Brunt ER, Klockgether T, Wullner U (2003) Gene expression profiling in ataxin3 -expressing cell lines reveals distinct effects of normal and mutant ataxin-3. J Neuropathol Exp Neurol 62:1006-1018.

Freiman RN, Tjian R (2003) Regulating the regulators: lysine modifications make their mark. Cell 112:11-17.

Gauthier LR, Charrin BC, Borrell-Pages M, Dompierre JP, Rangone H, Cordelieres FP, De Mey J, MacDonald ME, Lessmann V, Humbert S, Saudou F (2004) Huntingtin controls neurotrophic support and survival of neurons by enhancing BDNF vesicular transport along microtubules. Cell 118:127-138

Gervais FG, Singaraja R, Xanthoudakis S, Gutekunst CA, Leavitt BR, Metzler M, Hackam AS, Tam J, Vaillancourt JP, Houtzager V, Rasper DM, Roy S,
Hayden MR, Nicholson DW (2002) Recruitment and activation of caspase- 8 by the Huntingtin-interacting protein Hip-1 and a novel partner Hippi. Nat Cell Biol 4:95-105.

Guenther MG, Barak O, Lazar MA (2001) The SMRT and N-CoR corepressors are activating cofactors for histone deacetylase 3. Mol Cell Biol 21:6091-6101.

Haacke A, Broadley SA, Boteva R, Tzvetkov N, Hartl FU, Breuer P (2006) Proteolytic cleavage of polyglutamine-expanded ataxin-3 is critical for aggregation and sequestration of non-expanded ataxin-3. Hum Mol Genet 15:555-568.

Han X, Boyd PJ, Colgan S, Madri JA, Haas TL (2003) Transcriptional upregulation of endothelial cell matrix metalloproteinase-2 in response to extracellular cues involves GATA-2. J Biol Chem 278:47785-47791.

Hayakawa F, Towatari M, Ozawa Y, Tomita A, Privalsky M, Saito H (2004) Functional regulation of GATA-2 by acetylation. J Leukoc Biol 75:529-540.

Hicke L (2001) Protein regulation by monoubiquitin. Nat Rev Mol Cell Biol 2:195-201.

Huang JM, Kim JD, Kim H, Kim J (2006) An improved cloning strategy for chromatin-immunoprecipitation-derived DNA fragments. Anal Biochem 356:145-147.

Kawaguchi Y, Okamoto T, Taniwaki M, Aizawa M, Inoue M, Katayama S, Kawakami H, Nakamura S, Nishimura M, Akiguchi I (1994) CAG expansions in a novel gene for Machado-Joseph disease at chromosome 14q32.1. Nat Genet 8:221-228.

Kegel KB, Meloni AR, Yi Y, Kim YJ, Doyle E, Cuiffo BG, Sapp E, Wang Y, Qin ZH, Chen JD, Nevins JR, Aronin N, DiFiglia M (2002) Huntingtin is present in the nucleus, interacts with the transcriptional corepressor C-terminal binding protein, and represses transcription. J Biol Chem 277:7466-7476.

Kutney SN, Hong R, Macfarlan T, Chakravarti D (2004) A signaling role of histone-binding proteins and INHAT subunits pp32 and Set/TAF-I $\beta$ in integrating chromatin hypoacetylation and transcriptional repression. J Biol Chem 279:30850-30855.

Landschulz WH, Johnson PF, McKnight SL (1988) The leucine zipper: a hypothetical structure common to a new class of DNA binding proteins. Science 240:1759-1764.

Li F, Macfarlan T, Pittman RN, Chakravarti D (2002) Ataxin-3 is a histonebinding protein with two independent transcriptional corepressor activities. J Biol Chem 277:45004-45012.

Li J, Wang J, Wang J, Nawaz Z, Liu JM, Qin J, Wong J (2000) Both corepressor proteins SMRT and $\mathrm{N}$-CoR exist in large protein complexes containing HDAC3. EMBO J 19:4342-4350.

Macfarlan T, Kutney S, Altman B, Montross R, Yu J, Chakravarti D (2005) Human THAP7 is a chromatin-associated, histone tail-binding protein that represses transcription via recruitment of HDAC3 and nuclear hormone receptor corepressor. J Biol Chem 280:7346-7358.

Mao Y, Senic-Matuglia F, Di Fiore PP, Polo S, Hodsdon ME, De Camilli P (2005) Deubiquitinating function of ataxin-3: insights from the solution structure of the Josephin domain. Proc Natl Acad Sci USA 102:12700-12705.

Matilla A, Koshy BT, Cummings CJ, Isobe T, Orr HT, Zoghbi HY (1997) The cerebellar leucine-rich acidic nuclear protein interacts with ataxin-1. Nature 389:974-978.

Muratani M, Tansey WP (2003) How the ubiquitin-proteasome system controls transcription. Nat Rev Mol Cell Biol 4:192-201.

Murphy K, Shimamura T, Bejcek BE (2001) Use of fluorescently labeled DNA and a scanner for electrophoretic mobility shift assays. Biotechniques 30:504-508.

Nagy L, Kao HY, Chakravarti D, Lin RJ, Hassig CA, Ayer DE, Schreiber SL, Evans RM (1997) Nuclear receptor repression mediated by a complex containing SMRT, mSin3A, and histone deacetylase. Cell 89:373-380.

Nicastro G, Menon RP, Masino L, Knowles PP, McDonald NQ, Pastore A (2005) The solution structure of the Josephin domain of ataxin-3: structural determinants for molecular recognition. Proc Natl Acad Sci USA 102:10493-10498.

Nishiyama C, Nishiyama M, Ito T, Masaki S, Masuoka N, Yamane H, Kitamura T, Ogawa H, Okumura K (2004) Functional analysis of PU.1 domains in monocyte-specific gene regulation. FEBS Lett 561:63-68.

Ozawa Y, Towatari M, Tsuzuki S, Hayakawa F, Maeda T, Miyata Y, Tanimoto M, Saito H (2001) Histone deacetylase 3 associates with and represses the transcription factor GATA-2. Blood 98:2116-2123.

Perissi V, Aggarwal A, Glass CK, Rose DW, Rosenfeld MG (2004) A core- 
pressor/coactivator exchange complex required for transcriptional activation by nuclear receptors and other regulated transcription factors. Cell 116:511-526.

Scheel H, Tomiuk S, Hofmann K (2003) Elucidation of ataxin-3 and ataxin-7 function by integrative bioinformatics. Hum Mol Genet 12:2845-2852.

Schmitt I, Evert BO, Khazneh H, Klockgether T, Wuellner U (2003) The human $M J D$ gene: genomic structure and functional characterization of the promoter region. Gene 314:81-88.

Schneider R, Bannister AJ, Weise C, Kouzarides T (2004) Direct binding of INHAT to $\mathrm{H} 3$ tails disrupted by modifications. J Biol Chem 279:23859-23862.

Scott GK, Daniel JC, Xiong X, Maki RA, Kabat D, Benz CC (1994) Binding of an ETS-related protein within the DNase I hypersensitive site of the HER2/neu promoter in human breast cancer cells. J Biol Chem 269:19848-19858.

Seo SB, McNamara P, Heo S, Turner A, Lane WS, Chakravarti D (2001) Regulation of histone acetylation and transcription by INHAT, a human cellular complex containing the set oncoprotein. Cell 104:119-130.

Seo SB, Macfarlan T, McNamara P, Hong R, Mukai Y, Heo S, Chakravarti D (2002) Regulation of histone acetylation and transcription by nuclear protein pp32, a subunit of the INHAT complex. J Biol Chem 277:14005-14010.

Steffan JS, Kazantsev A, Spasic-Boskovic O, Greenwald M, Zhu YZ, Gohler H, Wanker EE, Bates GP, Housman DE, Thompson LM (2000) The Huntington's disease protein interacts with $\mathrm{p} 53$ and CREB-binding protein and represses transcription. Proc Natl Acad Sci USA 97:6763-6768.

Strom AL, Forsgren L, Holmberg M (2005) A role for both wild-type and expanded ataxin-7 in transcriptional regulation. Neurobiol Dis 20:646-655.

Torchia J, Rose DW, Inostroza J, Kamei Y, Westin S, Glass CK, Rosenfeld MG (1997) The transcriptional coactivator $\mathrm{p} / \mathrm{CIP}$ binds CBP and mediates nuclear-receptor function. Nature 387:677-684.

Tsai CC, Kao HY, Mitzutani A, Banayo E, Rajan H, McKeown M, Evans RM (2004) Ataxin-1, a SCA1 neurodegenerative disorder protein, is func- tionally linked to the silencing mediator of retinoid and thyroid hormone receptors. Proc Natl Acad Sci USA 101:4047-4052.

Tsuda H, Jafar-Nejad H, Patel AJ, Sun Y, Chen HK, Rose MF, Venken KJ, Botas J, Orr HT, Bellen HJ, Zoghbi HY (2005) The AXH domain of ataxin-1 mediates neurodegeneration through its interaction with Gfi-1/ Senseless proteins. Cell 122:633-644.

Uchihara T, Fujigasaki H, Koyano S, Nakamura A, Yagishita S, Iwabuchi K (2001) Non-expanded polyglutamine proteins in intranuclear inclusions of hereditary ataxias-triple-labeling immunofluorescence study. Acta Neuropathol (Berl) 102:149-152.

Warrick JM, Morabito LM, Bilen J, Gordesky-Gold B, Faust LZ, Paulson HL, Bonini NM (2005) Ataxin-3 suppresses polyglutamine neurodegeneration in Drosophila by a ubiquitin-associated mechanism. Mol Cell 18:37-48.

Weinmann AS (2004) Novel ChIP-based strategies to uncover transcription factor target genes in the immune system. Nat Rev Immunol 4:381-386.

Yang Z, Wara-Aswapati N, Chen C, Tsukada J, Auron PE (2000) NF-IL6 $(\mathrm{C} / \mathrm{EBP} \beta)$ vigorously activates illb gene expression via a Spi-1 (PU.1) protein-protein tether. J Biol Chem 27528:21272-21277.

Yoon HG, Chan DW, Huang ZQ, Li J, Fondell JD, Qin J, Wong J (2003) Purification and functional characterization of the human N-CoR complex: the roles of HDAC3, TBL1, and TBLR1. EMBO J 22:1336-1346.

Zhang J, Guenther MG, Carthew RW, Lazar MA (1998) Proteasomal regulation of nuclear receptor corepressor-mediated repression. Genes Dev 12:1775-1780.

Zhang J, Kalkum M, Chait BT, Roeder RG (2002) The N-CoR-HDAC3 nuclear receptor corepressor complex inhibits the JNK pathway through the integral subunit GPS2. Mol Cell 9:611-623.

Zoghbi HY, Orr HT (2000) Glutamine repeats and neurodegeneration. Annu Rev Neurosci 23:217-247.

Zuccato C, Tartari M, Crotti A, Goffredo D, Valenza M, Conti L, Cataudella T, Leavitt BR, Hayden MR, Timmusk T, Rigamonti D, Cattaneo E (2003) Huntingtin interacts with REST/NRSF to modulate the transcription of NRSE-controlled neuronal genes. Nat Genet 35:76-83. 